\title{
EXPERIMENTAL INVESTIGATION OF BREAKING CRITERIA OF DEEPWATER WIND WAVES UNDER STRONG WIND ACTION
}

\author{
Sang Ho Oh ${ }^{a^{*}}$, Natsuki Mizutani ${ }^{b}$, Kyung-Duck Suh ${ }^{\mathrm{a}}$, Noriaki Hashimoto ${ }^{\mathrm{c}}$ \\ ${ }^{a}$ School of Civil, Urban, and Geosystem Engineering, Seoul National University, \\ Seoul 151-742, Korea \\ ${ }^{b}$ Department of Civil Engineering, Osaka Sangyo University, \\ Nakagaito, Daito 574-8530 Japan \\ ${ }^{c}$ School of Urban and Environmental Engineering, Kyushu University, \\ Fukuoka 812-8581 Japan
}




\begin{abstract}
The present study describes an experimental investigation of breaking criteria of deepwater wind waves under strong wind action. In a wind wave flume, waves were generated using different wind speeds and measured at different locations to obtain wave trains of no, intermittent, or frequent breaking. Water particle movement and free surface elevation were measured simultaneously using a PIV system and a wave gauge, respectively. For wind waves, not all the waves measured at a fixed location are breaking waves, and the breaking of a larger wave is not guaranteed. However, the larger the wave height, the larger the probability of breaking. In order to take as many breaking waves as possible for the cases of frequent breaking, we used the waves whose heights were close to the highest one-tenth wave height. The experimental results showed that the geometric or kinematic breaking criteria could not explain the occurrence of breaking of wind waves. On the other hand, the vertical acceleration beneath the wave crest was close to the previously suggested limit value, $-0.5 g$, when frequent breaking of large waves occurred, indicating that the dynamic breaking criterion would be good for discriminating breaking waves under a strong wind action.
\end{abstract}

Keywords: wave breaking criteria; particle image velocimetry; wind waves; laboratory experiment 


\section{Introduction}

Wave breaking is a key factor in many physical processes at the air-sea interface. Even though we have come to understand wave breaking more in recent decades, the mechanism governing the inception of wave breaking is still not well known. To predict the onset of wave breaking, many criteria have been suggested, but none of them has been universally accepted in practical applications.

In general, these criteria are categorized into three types: geometric, kinematic, and dynamic criteria. Many previous studies have reported that the geometric breaking criteria are not adequate as indicators of wave breaking because they are quite sensitive to wave modulation, fetch length [31], and directionality [36]. Recently studies on kinematic breaking criterion have received much attention with the introduction of particle image velocimetry (PIV) techniques. The PIV technique enables one to measure wave kinematics in the proximity of free surface, which was not achievable with conventional experimental methods. Using this property of PIV, some researchers investigated the kinematic breaking criterion of paddle-generated waves $[3,19,30]$. PIV studies on the breaking criteria of wind-generated waves, however, are very rare though the characteristics of this type of waves may be different from those of mechanically generated waves. Compared with the previous two breaking criteria, the dynamic breaking criterion has not been much examined, particularly through the observation of the acceleration field beneath wave surface, so a detailed experimental investigation for this criterion is required.

One of the reasons for the scarcity of PIV studies on wind waves might be 
the difficulty of capturing the image of wind-generated breaking waves at a fixed location. A general PIV technique for observing wave breaking of mechanically generated waves is to register the video image of an isolated breaker which is generated by the frequency focusing method [22]. By focusing dispersive wave components within a wave packet on a certain location, it is possible to make the waves break there constantly. In contrast, sufficiently developed wind waves tend to break intermittently and they do not break at a fixed location. Hence, a different experimental procedure must be set up to investigate the breaking criteria of wind waves using a PIV system, which is the main concern of the present study.

To examine the validity of a wave breaking criterion, we have to apply the criterion to both non-breaking and breaking waves. In the wind wave flume that we used, most of the large waves, whose length scale is beyond a certain level so that human eyes tend to follow their propagation along the flume, broke at the end of the test section under strong wind conditions. On the contrary, the waves that were observed at the midpoint of the test section under gentle wind conditions hardly broke, showing only occasional micro-scale breakers. However, not all the waves measured at a fixed location are breaking waves, even though the wave field is sufficiently developed so that wave breaking occurs frequently here and there. Moreover, it is not guaranteed that a larger wave will break. However, the probability of breaking should increase with the wave height, which, in turn, increases with wind speed and fetch length.

Taking this fact into consideration, we performed the PIV experiment for different wind and fetch conditions. Continuous video images of wind-generated waves were captured along with the simultaneous measurement of the water 
surface elevation. For each experimental condition, the measurement was carried out many times, and the wave samples whose heights were close to the highest one-tenth wave height, $H_{1 / 10}$, of the wave population were selected to examine the wave breaking criteria. A proper breaking criterion would show a noticeable difference in the wave breaking index among the test cases since the fraction of breaking waves to the total waves is different for different experimental conditions. Note that this study does not attempt to suggest a new wave breaking criterion. The main objective is to evaluate the existing wave breaking criteria by observing local wave characteristics.

This paper is organized in the following order. Previous researches on the three breaking criteria are briefly reviewed in Section 2. In Section 3, the experimental apparatus and procedure are explained in detail. The data processing techniques of the acquired PIV images and wave data are described in Section 4. Experimental results are presented in Section 5, which is followed by an overall discussion of the wave breaking criteria in Section 6. Finally, major conclusions are given in Section 7.

\section{Review of Previous Studies}

\subsection{Geometric breaking criterion}

The classical geometric breaking criterion describes the limiting wave steepness in terms of wave height, $H$, and wave period, $T$. By this criterion, wave breaking occurs if $H \geq \beta g T^{2}$. The theoretical value of $\beta$ for Stokes wave 
is 0.027 , as shown in Michel [16]. The observed values of $\beta$, at sea and laboratory tanks, however, revealed appreciable discrepancy with large scatterings. A number of laboratory experiments on wind-generated waves (e.g. [17]) as well as paddle-generated waves (e.g. [2]) has reported that $\beta \approx 0.020$ is the most common value, regardless of the wave generation mechanism. On the other hand, field observations [7,32] showed that waves break even with $\beta$ values one order of magnitude smaller than the theoretical one. The difference in $\beta$ between the theory and observations is often ascribed to crest instabilities which incite waves to immediate breaking. In addition, the large gap between the field and laboratory measurements is viewed as a consequence of the difference of fetch lengths [31].

Besides the wave steepness, Kjeldsen and Myrhaug [10] introduced several wave shape parameters to discuss wave breaking. These parameters are the crest front steepness, $\varepsilon$, crest rear steepness, $\delta$, vertical asymmetry, $\lambda$, and horizontal asymmetry, $\mu$, the definitions of which are given in Fig. 1. They reported that breaking inception accompanies an asymmetric deformation of wave form and suggested critical values for these parameters. Some researchers have used these wave parameters to discuss their applicability to breaking waves. Bonmarin [2] measured the degree of wave asymmetry quantitatively to find that wave asymmetry tends to increase as a wave approaches to breaking. The influence of wave directionality was examined by She et al. [23], who observed these parameters for three dimensional breaking waves and reported that $\varepsilon$ and $\lambda$ are dependent on the wave directionality whereas $\delta$ and $\mu$ are not sensitive to the directionality. Wu and Nepf [36] extended this study with the waves whose 
directional property is different from each other. They concluded that all the four parameters are not robust indicators for wave breaking because they vary over wide ranges depending on the wave directionality and the spectral shape.

\subsection{Kinematic breaking criterion}

The most common kinematic breaking criterion is the ratio of horizontal water particle velocity at the wave crest to the wave phase speed. This criterion predicts the onset of wave breaking if the ratio is greater than unity. It sounds in conception very logical, but considerable discrepancy exists among experimental studies. Some researchers reported a particle velocity higher than the phase speed at the moment of wave breaking while others observed substantially smaller particle velocities than the phase speed of breaking waves.

The kinematic breaking criterion has commonly been validated by employing flow visualization techniques. Melville and Rapp [15] used laser anemometry to measure particle velocity at the water surface. Their experiments did not support this criterion though they did not discuss this subject in detail. PIV studies for plunging breaking waves $[3,19]$ observed greater particle velocities than the phase speed at the tip of overturning jets. In contrast, She et al. [24] reported that the ratio of the crest particle velocity to the wave speed is close to but less than unity for both plunging and spilling type breaking waves. Similar results were reported by Stansell and MacFarlane [30] who concluded that this breaking criterion is far from a global predictor of wave breaking.

Meanwhile, researches on the kinematic breaking criterion of wind waves are relatively scarce, particularly for the waves under strong winds. Hwang et al. [8] 
used this criterion to detect breaking waves from the measured wave record and discussed the characteristics of wind-generated breaking waves. Peirson and Banner [18] captured video images of the thin upper layer of micro-scale breaking waves. Their experimental data showed a particle velocity smaller than the mean wave speed in this region. Other PIV measurements of wind waves [26,38] mainly examined the turbulence structures beneath the waves and did not discuss the breaking criterion. Until now, few researches investigated the kinematic breaking criterion of wind waves that involve distinct breaking occurrences.

\subsection{Dynamic breaking criterion}

The most common dynamic breaking criterion is related to the particle acceleration near the wave crest. It describes wave breaking as the excess of vertical particle acceleration beyond a certain limit. Several threshold values have been suggested based on theoretical or experimental investigations but a general agreement has not been established yet.

Phillips [20] supposed that waves break if the downward acceleration of water particles is greater than gravity, $g$. Longuet-Higgins [12] theoretically showed that the downward acceleration near the crest of a regular wave is equal to $0.5 \mathrm{~g}$ for wave breaking. Snyder et al [27] used this value to predict the whitecap appearance in the ocean. Later Longuet-Higgins [13] reported that the observable particle acceleration of the almost highest wave would not be greater than $-0.39 g$. Srokosz [29] proposed a similar value of $-0.4 \mathrm{~g}$ by reexamining the geometric breaking criterion of Ochi and Tsai [17]. Dawson et al. [4] suggested a further less 
threshold value of $-0.33 g$ by comparing the calculated probability of occurrence of breaking with the experimental results.

\section{Laboratory Experiment}

\subsection{Experimental Apparatus}

The experiment was carried out in the wind wave flume at National Institute for Land and Infrastructure Management (NILIM) in Japan. The plan view of the flume is shown in Fig. 2. The uniform test section is $1.5 \mathrm{~m}$ wide, $1.3 \mathrm{~m} \mathrm{high}$, and $28.5 \mathrm{~m}$ long. The sidewalls consist of glass plates and the top of the flume is covered with wood plates. Glass panes were installed on the tank bottom at five locations along the flume, through which a laser beam could be projected vertically upward from underneath the tank. Wind was generated by an axial fan driven by a $50 \mathrm{~kW}$ variable-speed motor at the upstream end of the flume. The wind then passes through guide vanes, a fine mesh screen, and honeycombs so that the wind velocity at the inlet section is quite uniform. At the inlet, a horizontal guide plate is provided, which can be adjusted vertically so as to be located at the water surface.

PIV images were captured using a Photron FASTCAM-net Charge Coupled Device $(\mathrm{CCD})$ camera $(512 \times 480$ pixel resolution) with a Canon PH6X8 Macro lens $(48 \mathrm{~mm} / \mathrm{f} 1.0)$. The CCD camera allowed us to obtain gray-scale 8-bit range images. A global electronic shutter inside the camera controlled the transfer rate of image frames. The maximum frame transfer rate of the camera in full resolution was $250 \mathrm{fps}$ whereas the images could be transferred at higher speed with a 
reduced resolution. The captured images were recorded in a built-in memory of the PIV system in real time and later downloaded to the hard disk of the host computer.

For the light source, a $6 \mathrm{~W}$ water-cooled argon-ion laser (Spectra-Physics Stabilite 2017) was used. The color of the laser was green and its wavelength was $488 \mathrm{~nm}$. The main body of the laser beam generator was located about $5 \mathrm{~m}$ apart from the wave flume and the laser light was carried to the desirable position by an optical cable. The laser beam released was formed into a shape of a sheet after passing a cylindrical lens. The thickness of the laser sheet, controlled by spherical optics, was kept constant at $5 \mathrm{~mm}$ at still water level during the experiment.

White polyvinyl chloride (PVC) particles (manufactured by Shinetsu Chemical Co.) were used as seed particles. The mean diameter and the specific gravity of the particle in water were $150 \mu \mathrm{m}$ and 1.20 , respectively.

Together with the PIV system, a capacitance-type wave gauge was used to measure water surface displacement. We installed the wave gauge so that its wire was placed at the center of the FOV (Field of View). In this manner, we could obtain wave data as well as PIV images at the same time. The simultaneous recording of the wave data allowed us to calculate the local wave phase speed, as will be described in Section 4.2. The initiation of PIV image and wave data acquisition was controlled by a synchronizer.

Meanwhile, we found that a wave gauge used for measuring mechanically generated waves was not satisfactory for observing wind-generated waves since it vibrated rapidly under wind action. In order to minimize this vibration, we separated the head amplifier, the primary origin of the vibration, from the wave 
gauge and laid it on the ceiling of the wave tank, as shown in Fig. 3(b). The vibration caused by wind drag almost disappeared by this improvement. Moreover, we increased the distance between the support and wires of the gauge from $5 \mathrm{~cm}$ to $20 \mathrm{~cm}$ so that the support was not located inside the FOV.

\subsection{Experimental Procedure}

\subsubsection{Preliminary experiment}

The preliminary experiment was performed at five locations, where the glass panes were positioned, with three different winds. The preliminary experiment was required to know the shape of vertical wind profiles and the characteristics of wave fields along the wind wave flume prior to conducting the main experiment. In the main experiment, reliable estimates of the wind and wave parameters could not be obtained because the measurement time was not long due to the restriction of the synchronized PIV system. Wind speeds were measured intensively in the proximity of air-water interface in order to accurately estimate the wind friction velocities, which have close relationship with the wind profiles near the water surface. The total number of measuring elevations varied from 35 to 40 within the range from the free surface up to $50 \mathrm{~cm}$ above the still water level, depending on experimental conditions. At each elevation, wind speeds were measured for 60 seconds at the sampling rate of $20 \mathrm{~Hz}$ to obtain the time-averaged wind velocity.

Wave measurements were made at the sampling rate of $200 \mathrm{~Hz}$ to obtain 131,200 data, and the first 131,072 data were subjected to spectral analysis. For the same experimental condition, wave measurements were made three times to examine the reproducibility of wave fields. The wave spectra of the three 
observations were almost same (the results are not shown), verifying the reproducibility of the wave field under the same wind condition.

\subsubsection{Main experiment}

The main experiment was conducted at two locations (stations $\mathrm{C}$ and $\mathrm{E}$ in Fig. 2), and three different winds were tested. The experimental conditions and parameters of the six test cases are summarized in Table 1. The wind and wave parameters in the table were calculated from the results of the preliminary experiment of the same experimental conditions. The values of wind friction velocity, $U_{*}$, were calculated by curve-fitting to a logarithmic wind profile. The values of $U$ and $U_{*}$ were slightly different depending on the fetches (e.g. Case 1 and 2) even though the wind input was the same. This difference is due to the calculation of the parameters using the measured wind profile at each fetch.

Fig. 3 is the schematic diagram of the experimental setup. In order to conduct the PIV experiment in daytime, a dark room was constructed at each location. In addition, a black thin plate was attached on the far sidewall of the flume to maximize the illumination of seed particles. The water depth was kept constant at $53 \mathrm{~cm}$ for all the tests, in which the spectral peak wave component always fell under the deep-water condition. The laser sheet was projected vertically upward along the centerline of the flume, parallel with the sidewalls. The wave gauge was setup for the wire to be located at the center of the FOV longitudinally and $3 \mathrm{~mm}$ away from the edge of the laser sheet laterally. In this manner, we could obtain synchronized wave and image data that had almost identical wave properties while the wire scarcely appeared in the captured images. The camera was 
positioned below the wave trough level and fixed so that its angle was $20^{\circ}$ upward. With a smaller angle, the waves on the near sidewall intermittently masked those on the far side of the flume since the wave phases were not always identical in the lateral direction. The maximum imaging error in vertical scale due to this photographing distortion was estimated to be $5 \%$.

In order to compute the magnification factor of the PIV images, a grid paper was photographed while the wind fan was not in operation. The FOV of the camera was $23.3 \mathrm{~cm}$ wide and $21.8 \mathrm{~cm}$ high for all the test cases, and the corresponding image resolution was $0.455 \mathrm{~mm} / \mathrm{px}$. After photographing the grid paper, wind was generated over quiescent water. To permit the slower traveling high-frequency component waves to travel to the measuring location, a sufficient waiting time was allowed to elapse after the initiation of wind generation. Seed particles were spread into water by hand about 20 seconds before recording PIV images and added repeatedly before each run. The sampling rate of the wave measurement was $200 \mathrm{~Hz}$ for all the test cases, whereas the frame transfer rate and shutter speed of the CCD camera varied depending on the experimental conditions as shown in Table 1. Thus, the total time of data acquisition was also different for each case due to the limit of the memory capacity: the faster the frame transfer rate, the shorter the observation time. The camera registered wave images at a constant frame transfer rate and shutter speed, and no frame-straddling technique was used. A total of 2184 PIV images was captured continuously and stored in a built-in memory temporarily and then transferred to the host computer. The data acquisition was repeated eight times for the first two cases and 15 times for others.

\section{Data Processing}




\subsection{Basic analysis}

The wave data were analyzed by zero-downcrossing method and separated into individual waves. Studies on wave breaking frequently employed zerodowncrossing method rather than zero-upcrossing method because a wave profile between two consecutive zero-downcrossing points contains a complete wave front, which is physically more relevant to wave breaking [7]. Table 1 shows the total number of observed waves, and the highest one-tenth wave height and period of each test case.

The acquired images were analyzed by a PIV analysis software, INSIGHT (TSI Inc.). The software computes the velocities of a captured flow field by estimating particle displacements between two consecutive frames with FFTbased cross-correlation peak search algorithm [9]. The background noise of PIV images was removed prior to the analysis. The size of an interrogation window was $32 \times 32$ pixels and the window was overlapped by 16 pixels with neighboring ones. This produces a $31 \times 29$ array of velocity vector maps for each PIV image, the resolution of which was $7.3 \times 7.3 \mathrm{~mm}^{2}$. The raw velocity vector matrix calculated by the software was further subjected to post-processing in which spurious vectors involved were eliminated to obtain a better flow field. The postprocessing was done by using a computer program constructed by the authors; the built-in tools provided by the INSIGHT were not used.

Then the wave samples were selected to examine the wave breaking criteria. All the waves separated by the zero-crossing analysis were sorted by wave height 
and 20 waves whose heights were close to $H_{1 / 10}$ were selected. The number of wave samples was set to be 20 so that the number of samples did not exceed $10 \%$ of the total number of waves of Case 6, which was the least in this experiment. Synchronously acquired PIV images of the selected wave samples were also picked up from the image data.

In order to obtain a rough estimate of the probability of wave breaking, the fraction of breaking waves, or the ratio of breaking waves to the total observed waves, was calculated for each test case by examining the video images with eyes and is shown in Table 1. The waves that show apparent spilling wave crest or whitecaps were regarded as the breaking waves. A sample image of the spilling breaker is shown in Fig. 4. The fraction of wave breaking increases as wind and fetch becomes stronger and longer, respectively, as shown in Table 1. The result of the present study is generally consistent with those of previous laboratory experiments $[8,37]$.

\subsection{Calculation of phase speed}

The wave phase speed must be calculated to examine the kinematic breaking criterion. The simplest formulation to calculate the wave phase speed is to use the dispersion relationship of the linear wave theory. By this formulation, the wave phase speed is non-local along a wave cycle. In the present study, the local phase speed was calculated empirically by correlating the PIV images with the synchronously observed wave record as described below. First, the wave surface profile of a given image was found by examining the neighboring images. Since the wave gauge wire was positioned at the center of an image as shown in Fig. 
3(b), the surface at the center of the image must be equal to the surface elevation measured by the wave gauge. If we assume a wave travels without change of its shape during a short time interval and suppose a certain phase speed, then it is possible to obtain a surface line using the synchronously measured wave record. That is, the surface points at the center of previous images, measured by the wave gauge, correspond to those at the front side of the present image. Similarly, the surface points at the back side of the wave correspond to those at the center of subsequent images. Then we can determine the phase speed that provides the surface line closest to the observed surface profile by trial and error.

The above method was applied for the calculation of local phase speeds at a wave crest and trough. Fig. 5 shows the phase speeds of the 20 selected waves of the six cases at their respective wave crests and troughs. The straight lines indicate the phase speed calculated by the linear dispersion relationship. The measured phase speeds are larger than those predicted by the linear wave theory. It has been reported that the phase speed is greater than the value predicted by the linear wave theory when wind action is present [25,35]. Also found in the figure are larger phase speeds at wave crests than at wave troughs. On the average, the phase speed was about $25 \%$ greater than that predicted by the linear dispersion relationship.

It would be too laborious to find the local phase speed for all the images using the above method. Hence, at other phases of a wave, excluding the crest and trough of a wave, the phase speed was calculated by using an empirical formula:

$$
c=\frac{g T}{2 \pi}+\alpha(\phi) U
$$


where $\alpha$ is a function of the local phase function, $\phi$, which varies from 0 to $2 \pi$ between two zero-downcrossings and is expressed as

$$
\alpha(\phi)=\frac{\alpha_{c}+\alpha_{t}}{2}-\frac{\alpha_{c}-\alpha_{t}}{2} \sin \phi
$$

where $\alpha_{c}$ and $\alpha_{t}$ are the mean of $\alpha$ at the wave crest and trough, respectively, and $U$ is the free-stream wind speed measured at $40 \mathrm{~cm}$ above the still water level in this experiment. The values of $\alpha_{c}$ and $\alpha_{t}$ were determined by linear regression with the 20 wave samples of each test case. Table 1 lists these parameter values. In the table also shown is $\alpha_{m}$, which is the average of $\alpha_{c}$ and $\alpha_{t}$ and can be regarded as $\alpha$ at the mean water level.

The values of $\alpha_{m}$ correspond to the results of previous researches. The wave periods of the present data were distributed from 0.2 to $0.7 \mathrm{~s}$, for which $\alpha$ ranges approximately from 0.01 to 0.02 according to Shemdin [25]. The data of Wright and Keller [34], obtained from radar backscattering of high frequency waves, gives larger values of $\alpha$, in the range of 0.025 to 0.030 .

\subsection{Vector editing}

Incorrect vectors of the raw velocity field, arising from the wrong evaluation of correlation-peak displacements, were automatically detected and replaced by alternative values. First, the raw velocity vectors were subjected to a global filter, which eliminates all the vectors whose magnitudes were not within a certain limit. The limit value, which was found based on the global histogram plots as shown in 
Raffel et al. [21], was determined differently depending on the test cases.

Second, a local median filter detects remaining spurious vectors by comparing each velocity vector with a limit value computed from eight surrounding vectors. Westerweel [33] showed that the local median filter is the most efficient in detecting spurious vectors in the PIV data. However, in the midst of the data processing, it was found that the median filter of Westerweel [33] is not efficient in detecting error vectors that are comparable with neighboring vectors in magnitude but deviate largely in direction. This is because the conventional median filter considers only a vector's magnitude, as already pointed out by Liang et al. [11]. Hence, a median filter that considers both direction and magnitude of velocity vectors would be better in detecting erroneous vectors. In the present study, a velocity vector was regarded as valid if it satisfied the following criteria:

$$
\begin{aligned}
& |\mathbf{v}|_{\text {median }}-C_{1} \times|\mathbf{v}|_{\text {rms }} \leq|\mathbf{v}| \leq|\mathbf{v}|_{\text {median }}+C_{1} \times|\mathbf{v}|_{\text {rms }} \\
& \theta_{\text {median }}-C_{2} \times \theta_{\text {rms }} \leq \theta \leq \theta_{\text {median }}+C_{2} \times \theta_{\text {rms }}
\end{aligned}
$$

where $|\mathbf{v}|$ and $\theta$ are the magnitude and angle of a velocity vector and the coefficients $C_{1}$ and $C_{2}$, which are defined as a function of the relative depth, $k z$, are expressed as

$$
C_{1}=\left\{\begin{array}{clc}
0.3-0.15 \mathrm{kz} / \mathrm{kz} \text { surf }, & \text { if } & 0<k z \\
0.3-0.3 \mathrm{kz} / \pi, & \text { if } & -\pi<k z \leq 0 \\
0.6, & \text { if } & k z \leq-\pi
\end{array}\right.
$$


$C_{2}=\left\{\begin{array}{clc}0.5-0.25 k z / k z_{\text {surf }}, & \text { if } & 0<k z \\ 0.5-0.5 k z / \pi, & \text { if } & -\pi<k z \leq 0 \\ 1.0, & \text { if } & k z \leq-\pi\end{array}\right.$

where $k$ is the wave number, $z$ is the vertical coordinate measured vertically upward from still water line, and $z_{\text {surf }}$ is the water surface elevation. The above filtering criteria depending on the relative depth, $k z$, were determined on the basis of a simple numerical test and showed good performance in detecting erroneous vectors without influencing correct vectors.

Special care was given to the velocity vectors right beneath the water surface. There were fewer than eight neighboring vectors, and the median filter might give skewed criteria, probably of smaller magnitude. To cope with this exceptionality, the topmost vectors of the velocity matrix were checked columnwise with another limit value. To obtain the limit value, the velocity vectors underneath the topmost vector were extrapolated using the exponential fitting function based on the linear wave theory. If the magnitude of the topmost vector was less than 0.75 times the calculated value, the vector was replaced by the extrapolated one. At other exceptional points such as image corners or boundaries, where the number of neighboring vectors was also fewer than eight, this method was not applied because the magnitude of the vector was not significant there.

Finally, all the detected spurious vectors were replaced by new vectors calculated by weight-averaging the eight surrounding vectors of a $3 \times 3$ window: 
$\mathbf{v}_{\text {new }}=\frac{1}{N} \sum_{n=1}^{N} \mathbf{v}_{n} r^{-2}$

where $N$ is the number of surrounding vectors, $\mathbf{v}_{n}$, whose relative distance from the center of the window is given by $r$. In general, the number of corrected spurious vectors was less than $3 \%$ of the total number of velocity vectors. No further correction was used to smooth the resulting vector field. Fig. 6 shows a snapshot of a PIV image together with the corresponding velocity and acceleration maps that were obtained by the above procedure. The acceleration field was calculated by finite differentiating the velocity field using Eq. (10) shown in Section 5.3.

\subsection{Error Analysis}

The uncertainty of measurement of the PIV data was investigated in terms of random errors and bias errors. Raffel et al. [21] showed the Monte-Carlo simulation results of the RMS random error with respect to the particle image diameter, displacement, and density. In the present PIV experiment, the diameter of particle image was approximately three pixels and the corresponding RMS uncertainty for $32 \times 32$ interrogation window was about 0.03 pixels. Similarly, the particle image density, 20 on the average for all the test cases, leads to the RMS error of 0.02 pixels. The RMS error due to the maximum particle image shift, which is about 10 near the wave crest, is 0.05 pixels. The combined error for these sources of error can be estimated by calculating the square root of the sum of the squared errors [5], and was estimated to be 0.062 pixels. This value indicates that 
the expected total RMS error is no greater than $0.62 \%$ near the wave crest region.

On the other hand, the bias error due to the velocity gradient was estimated by applying a condition for the optimal PIV setup suggested by Keane and Adrian [9]. For a double-frame single-exposure system, we could estimate an upper bound of the velocity gradient by the following inequality:

$$
|\Delta u|<\frac{0.03 d_{I}}{M \Delta t}
$$

where $d_{I}$ is the size of interrogation region in the pixel, $M$ is the magnification factor, and $\Delta t$ is the time interval between light pulses, or the inverse of frame transfer rate. The computed upper bounds of a velocity gradient, $|\Delta u|$, are 0.109 , 0.055 , and $0.026 \mathrm{~m} / \mathrm{s}$ for the frame transfer rate of 250,125 , and $60 \mathrm{fps}$, respectively. All the experimental data fall within these limits, so the bias error of the present PIV system can be considered negligible.

\section{Results}

\subsection{Geometric breaking criterion}

The mean values of the five wave parameters that are related to wave geometry are plotted in Fig. 7. The horizontal dashed line shown in Fig. 7(a) denotes the limit wave steepness, $H / g T^{2}=0.020$ whereas the dashed lines in Figs. 7(b) to (d) are the upper and lower limits of the breaking occurrence reported by Kjeldsen and Myrhaug [10]. The limits of $\mu$ are not shown in Fig. 
7(e) since it is far above the parameter values of the present data. The error bars shown in these figures indicate the $95 \%$ confidence intervals. $\varepsilon$ and $\delta$ were computed using the wavelength due to the linear wave theory to compare with previous researches.

The value of $H / g T^{2}$ ranged from 0.0197 to 0.0248 , which is slightly greater than the common limit value, 0.02. It was greater than the limit value even in Case 1, where no breaking waves were observed. Similarly, all the values of $\varepsilon$, $\delta$, and $\lambda$ of the six cases lay in the ranges of breaking occurrence and showed little discrepancy between test cases, irrespective of their significant difference in the fraction of breaking waves as shown in Table 1 . This clearly shows that the geometric breaking criteria are insufficient in explaining the occurrence of wave breaking. The values of $\mu$ shown in Fig. 7(e) are quite different from the values given by Kjeldsen and Myrhaug [10], $\mu=[0.84,0.95]$. The significant difference in $\mu$ from the present study might be due to the use of the mean water level (MWL) in their study.

\subsection{Kinematic breaking criterion}

In order to examine the kinematic breaking criterion, the horizontal particle velocity at the water surface was calculated by extrapolating the velocities underneath the surface. The surface velocity at an image center, $u_{s}$, is divided by the local wave phase speed, $c$, calculated by the method described in Section 4.2. Fig. 8 shows the values of $u_{s} / c$ of the 20 selected waves of the six test cases, as a function of normalized time, $t / T$, where $T$ is the wave period. Note that the 
number of data points is different among the figures since the number of PIV images within a wave cycle is not the same due to different time scales and frame transfer rates. As seen in the figures, the values of $u_{s} / c$ near wave crests, or at $t / T=0.75$, are substantially less than unity. Even in Case 6, where the probability of breaking occurrence is the highest, the maximum of $u_{s} / c$ is less than 0.75 . Hence, the kinematic breaking criterion is not satisfied for all the cases.

Fig. 9 compares the means of $u_{s} / c$ for the six cases shown in Fig. 8. The mean values of $u_{s} / c$ at the wave crest increase monotonously from Case 1 to 6 . This suggests that the values of $u_{s}$ at the crest increase more rapidly than $c$ as wind and fetch length increase. In contrast, the values of $u_{s} / c$ at the wave trough do not show noticeable discrepancy among the test cases, except Case 1 . In general, the magnitude of $u_{s} / c$ at wave crests is approximately double of that at wave troughs for all the cases.

Meanwhile, the normalized mean velocity field was calculated by dividing the velocity by the corresponding wave phase speed and ensemble-averaging them:

$$
<u / c>=\frac{1}{20} \sum_{i=1}^{20} u_{i}(x, z, t) / c_{i}
$$

where $\langle\cdot>$ denotes the ensemble average. Fig. 10 shows the vertical profiles of $<u / c\rangle$ underneath the wave trough and crest as a function of normalized depth, $z / H_{1 / 10}$. As in Fig. $9,\langle u / c\rangle$ at the crest are far less than unity. The velocity 
profiles are close to one another in the range of $z / H_{1 / 10}<0$. Above this range, they start deviation, showing in general, larger values of $\langle u / c\rangle$ for larger waves. Similar trend is found beneath the wave trough, i.e., larger scatterings near the surface and larger $\langle u / c\rangle$ for larger waves.

The dotted lines in Fig. 10 are the values of $u / c$, where $u$ is the particle velocity calculated by the linear wave theory and $c$ is the phase speed calculated by Eq. (1). Near the water surface, under both wave crest and trough, the measured particle velocity is somewhat smaller than the theoretical value, the reason of which is unknown.

\subsection{Dynamic breaking criterion}

The validity of the dynamic breaking criterion is examined in a manner similar to the previous section. The instantaneous acceleration field was obtained by using the finite differentiation scheme shown in Son and Kihm [28]:

$$
\begin{gathered}
\frac{D u\left(x_{0}, z_{0}, t_{0}\right)}{D t}=\frac{1}{2}\left[\frac{u\left(x_{0}, z_{0}, t_{1}\right)-u\left(x_{0}, z_{0}, t_{0}\right)}{\Delta t}+\frac{u\left(x_{0}, z_{0}, t_{0}\right)-u\left(x_{0}, z_{0}, t_{-1}\right)}{\Delta t}\right] \\
+\frac{1}{2} u\left(x_{0}, z_{0}, t_{0}\right)\left[\frac{u\left(x_{1}, z_{0}, t_{0}\right)-u\left(x_{0}, z_{0}, t_{0}\right)}{\Delta x}+\frac{u\left(x_{0}, z_{0}, t_{0}\right)-u\left(x_{-1}, z_{0}, t_{0}\right)}{\Delta x}\right] \\
+\frac{1}{2} w\left(x_{0}, z_{0}, t_{0}\right)\left[\frac{u\left(x_{0}, z_{1}, t_{0}\right)-u\left(x_{0}, z_{0}, t_{0}\right)}{\Delta z}+\frac{u\left(x_{0}, z_{0}, t_{0}\right)-u\left(x_{-1}, z_{-1}, t_{0}\right)}{\Delta z}\right]
\end{gathered}
$$

In the differentiation, the time interval of the local acceleration term was set to be almost constant to minimize the influence of different frame transfer rates among the test cases. That is, we used the images before and after the present time $\left(t_{0}\right)$ by 
one frame for Cases 1 and 2 , where $\Delta t=t_{1}-t_{0}=t_{0}-t_{-1}=1 / 60 \approx 0.0167 \mathrm{~s}$. For Cases 3 and 4, the images before and after the present image by two frames were used, or $\Delta t=2 / 125=0.016 \mathrm{~s}$. Similarly, $\Delta t=4 / 250=0.016 \mathrm{~s}$ for Cases 5 and 6. It was found that the contribution of the convective acceleration term to the total acceleration was marginal (the results are not shown).

Figs. 11(a) to (f) show the normalized vertical accelerations at the water surface, $a_{s} / g$, of the 20 selected waves as a function of $t / T$. Compared with the values in Fig. 8, the values of $a_{s} / g$ in Figs. 11(a) to (f) show more scattering as a result of the differentiation. In general, the variation of $a_{s} / g$ with $t / T$ shows a similar trend in all the six cases. The value of $\left|a_{s} / g\right|$ at wave crests $(t / T \approx 0.75)$ shows an increasing trend from Case 1 to 6 . For some data of Cases 5 and $6,\left|a_{s} / g\right|$ is close to unity.

The mean values of $a_{s} / g$ of the six test cases are compared in Fig. 12. The minimum values of $a_{s} / g$ for Cases 1 to 3 are below the limit value -0.5 suggested by Longuet-Higgins [12] and show distinct discrepancy among them whereas those of Case 4 to 6 are slightly below the limit value.

As with the mean velocity field, the calculation of the normalized mean acceleration field was carried out by ensemble-averaging the differentiated velocity maps of the selected waves:

$<a / g>=\frac{1}{20} \sum_{i=1}^{20} \frac{1}{g} \frac{D w(x, z, t)_{i}}{D t}$ 
Fig. 13 shows the vertical profiles of $\langle a / g\rangle$ underneath the wave trough and crest as a function of $z / H_{1 / 10}$. It is observed that the vertical profiles form a line, particularly in the range of $z / H_{1 / 10}<-1$. Above this range, the profiles of Cases 3 to 6 still follow the line and those of Cases 1 and 2 deviate a little from the line. This indicates that the vertical acceleration might have a functional relationship with the vertical coordinate normalized by the representative wave height. Note that the vertical profiles of acceleration show less scattering than the velocity profiles shown in Fig 10.

The measured vertical acceleration profiles are compared with the theoretical ones of Longuet-Higgins [14] in Fig. 13. The acceleration profiles of linear wave theory were not used because they are not relevant to the Lagrangian motion of water particles. As shown in Fig. 13, the measured accelerations are relatively well predicted by the theory of Longuet-Higgins [14]. One noteworthy thing is that the rate of increase with elevation of the theoretical acceleration slightly decreases near the wave crest. This trend is also seen in the measured acceleration profiles.

\subsection{Dependence on wind speed and fetch length}

It seems that the wave breaking criteria explained in Section 2 might have a relationship with the wind speed or fetch length. In order to examine this, the mean values of the wave breaking criteria were plotted as a function of the inverse wave age, $U_{*} / c_{p}$, in Fig. 14. As in Fig. 7, the error bars indicate the $95 \%$ confidence intervals and the dashed lines denote the respective limit values of the 
breaking occurrence. The values of $u_{s} / c$ and $a_{s} / g$ in the figure correspond to the values at the wave crest. $U_{*} / c_{p}$ represents the relative magnitude of wind speed to the wave phase speed and is often interpreted as the wind forcing to underlying waves. The values of $U_{*} / c_{p}$ is given in Table 1 . The values of $U_{*} / c_{p}$ for Cases 1 and 4 are almost the same, implying that the degree of wind forcing is almost the same for the two cases.

The first five parameters in Fig. 14 gradually increase with $U_{*} / c_{p}$ at smaller values and become constant at larger values, while the last one, $a_{s} / g$, monotonously decreases with $U_{*} / c_{p}$. The values of $u_{s} / c$ show an increasing trend on the whole, but the fluctuation was comparatively large. It seems that wind forcing has some influence on the variation of these parameters, particularly when $U_{*} / c_{p}<0.8$, implying that the effects of wind forcing are more significant when $U_{*} / c_{p}$ is relatively low. In addition, the increasing trend of $\varepsilon$ and $\delta$ as well as $\mu$ shows that the wind effects are more prominent for increasing the crest height $\eta^{\prime}$ rather than the wavelength or wave height.

Similarly, the relationship between the wave breaking criteria and the fetch length was examined. Fig. 15 shows the values of the seven parameters as a function of the nondimensional fetch, $g F / U_{*}^{2}$, which are given in Table 1. Compared to the previous figure, the fluctuation of the parameters with $g F / U_{*}^{2}$ was much less. The first six parameters gradually decrease with the nondimensional fetch, whereas the last one shows an increasing trend. This implies that the possibility of breaking occurrence is reduced with the 
nondimensional fetch. However, this fact may not be interpreted as a general trend because the range of the nondimensional fetch was very narrow and confined to very young waves.

\section{Discussion}

As found in the previous studies, the present study also showed that the geometric breaking criterion depending on the local wave shape is not a good indicator of wave breaking. Neither wave steepness nor asymmetry seems to be suitable as a breaking criterion.

The kinematic breaking criterion seems to be the most sensitive to the change of experimental conditions. However, the overall values of $u_{s} / c$ of the present data are far below the limiting value of unity. It seems that wave breaking could occur even when this breaking criterion was not satisfied, as Stansell and MacFarlane [30] pointed out. In particular, this breaking criterion would not be satisfied for wind-generated waves because the phase speed significantly increases as a consequence of wind forcing. The water particle velocity right beneath water surface also may increase due to the wind-induced surface drifts. Peirson and Banner [18], however, reported that the particle velocity underneath the surface was much less than the mean wave speed at wave breaking, though their study was concerned with tangential stress underneath micro-scale wave breaking. The present study did not attempt to estimate water particle velocities in the thin layer right beneath the surface. The image resolution of the present PIV experiment was about $7 \mathrm{~mm}$, which was not sufficient to measure the particle velocity at the subsurface. 
The disagreement in the literature regarding the kinematic breaking criterion might be partly due to the inconsistency of estimating the water particle velocity and the wave phase speed. The essential point with respect to this criterion is the correct estimation of the two quantities because it is simply expressed as a ratio between them. The correct estimation of the phase speed is more important since we can obtain reliable particle velocity estimates near the surface by using visualization techniques, such as PIV. The use of dispersion relationship or the Hilbert transform is not adequate for calculating the phase speed of wind waves. As stated previously, when wind action is present, the phase velocity is greater than that by the linear wave theory. The Hilbert transform is intrinsically applicable only for narrow-banded wave processes, the condition of which is hardly satisfied for wind-generated waves that involve high-frequency oscillations.

In the present study, the wave phase speed was calculated empirically as explained in Section 4.2. The basic assumption of this method is that a wave travels without the change of its form during a short time interval. This assumption was required because the local phase speed was estimated by using the wave data measured at a single location. If the local phase speed was directly measured with more than two wave gauges spaced closely, this assumption would not be necessary. In this situation, the above method can be applied for automatic detection of the water surface in a PIV image by linking the wave elevations measured by the wave gauges.

The values of $a_{s} / g$ at the wave crest for wave breaking of the present study were similar to the previously proposed limit values: $a_{s} / g \approx-0.5$ for Cases 4 to 6 , in which most of the large waves were expected to break. This 
similarity suggests that the dynamic breaking criterion is not greatly affected by winds and can be used universally regardless of the presence of wind action. In general, the magnitude of $a_{s} / g$ increases with wind speed and fetch length, though prominent discrepancy was not found among the values of Case 4 to 6 .

One important aspect regarding particle acceleration is the difference between the Eulerian and Lagrangian acceleration. Longuet-Higgins [13,14] showed that the accelerations estimated by the two different reference frames could result in significantly different values. The acceleration calculated by the differentiation of a PIV image pair is regarded as an Eulerian quantity even though it follows a particle displacement during short time intervals [6]. Hence the examination of the dynamic breaking criterion using PIV measurements should be viewed in this respect. However, the acceleration calculated by PIV would involve less uncertainty than that calculated by double differentiation of the water surface displacement.

All the wave breaking criteria discussed in this study are based on the local wave property. In contrast, there exists another type of wave breaking criteria that are related to global property of waves. These involve the global wave steepness, higher harmonic energy evolution [22], and momentum and energy growth rate [1]. These parameters were not considered in the present study. If wave breaking is associated with wave spectral variation, these parameters would be more promising for detecting the occurrence of wave breaking.

\section{Conclusions}

The wave breaking criteria of wind waves were investigated by conducting a 
PIV experiment in a wind wave flume. Simultaneous measurements of water particle movement and water surface elevation of wind-generated waves enabled us to examine three different types of wave breaking criteria. The wave breaking criteria were examined for different wind and fetch conditions, which represent different probability of wave breaking.

We found that the local wave shape parameters related to geometric breaking criteria are not closely related to the probability of wave breaking, implying that they are not good indicators of wave breaking. As for the kinematic criterion, the ratio of horizontal particle velocity to the local wave phase speed at the wave crest was substantially less than unity, even for the test case where most of the large waves break, indicating that the kinematic breaking criterion is not good either. The vertical acceleration beneath the wave crest of the present data was close to the previously suggested limit value, $-0.5 g$, when breaking waves were prevailing in a wave field. Thus the dynamic breaking criterion seems to be applicable in the presence of wind action. The dynamic criterion, which did not receive sufficient investigation due to the difficulty of measurement of acceleration, should be investigated more intensively in the future.

\section{Acknowledgements}

This research was supported by the Brain Korea 21 project. The first author was partly supported by the Port and Airport Research Institute (PARI) during his visit to Japan. 


\section{REFERENCES}

1. Banner M.L., Tian X. On the determination of the onset of breaking for modulating surface gravity water waves. J Fluid Mech 1998; 367:107-137.

2. Bonmarin P. Geometric properties of deep-water breaking waves. J Fluid Mech 1989;209:405-433.

3. Chang K.-A., Liu P.L.-F. Velocity, acceleration and vorticity under a breaking wave. Phys Fluids 1998;10(1):327-329.

4. Dawson T.H., Kriebel D.L., Wallendorf L.A. Breaking waves in laboratorygenerated JONSWAP seas. Appl Ocean Res 1993;15:85-93.

5. Dong P., Hsu T.-Y., Atsavapranee P., Wei T. Digital particle image accelerometry. Exp Fluids 2001;30:626-632.

6. Jakobsen M.L., Dewhirst T.P., Greated C.A. Particle image velocimetry for predictions of acceleration fields and force within fluid flows. Meas Sci Technol 1997;8:1502-1516.

7. Holthuijsen L.H., Herbers T.H.C. Statistics of breaking waves observed as whitecaps in the opensea. J Phys Oceanogr 1986;16:290-297.

8. Hwang P.A., Xu D., Wu J. Breaking of wind-generated waves: measurements and characteristics. J Fluid Mech 1989;202:177-200.

9. Keane R.D., Adrian R.D. Theory of cross-correlation analysis of PIV images. In: Nieuwstadt F.T.M., editor. Flow visualization and image analysis. Kluwer academic publishers, 1993. p. 1-25.

10. Kjeldsen S.P., Myrhaug D. Wave-wave interactions, current-wave interactions and resulting extreme waves and breaking waves. In: Proceedings of the 17th International Conference on Coastal Engineering, Sydney, Australia, 1980. p. 2277-2303.

11. Liang D.F., Jiang C.B., Li Y.L. Cellular neural network to detect spurious vectors in PIV data. Exp Fluids 2003;34:52-62.

12. Longuet-Higgins M.S. The generation of capillary waves by steep gravity waves. J Fluid Mech 1963;16:138-159.

13. Longuet-Higgins M.S. Acceleration in steep gravity waves. J Phys Oceanogr 1985;15:1570-1579.

14. Longuet-Higgins M.S. Eulerian and Lagrangian aspects of surface waves. J Fluid Mech 1986;173:683-707. 
15. Melvill W.K., Rapp R.J. The surface velocity field in steep and breaking waves. J Fluid Mech 1988;189:1-22.

16. Michel J.H. The highest wave in water. Philos Mag 1893;36:430-437.

17. Ochi M.K., Tsai C.H. Prediction of occurrence of breaking waves in deep water. J Phys Oceanogr 1983;13:2009-2019.

18. Peirson W.L., Banner M.L. Aqueous surface layer flows induced by microscale breaking wind waves. J Fluid Mech 2003;479:1-38.

19. Perlin M., He J., Bernal L.P. An experimental study of deep water plunging breakers. Phys Fluids 1996;8:2365-2374.

20. Phillips O.M. The equilibrium range in the spectrum of wind-generated waves. J Fluid Mech 1958;4:785-790.

21. Raffel M., Willert C.E., Kompenhans J. Particle image velocimetry. Springer, 1998.

22. Rapp R.J., Melville W.K. Laboratory measurements of deep water breaking waves. Philos Trans R Soc London 1990;331A:735-800.

23. She K., Greated C.A., Easson W.J. Experimental study of three-dimensional wave breaking. J Waterw Port Coastal Ocean Eng 1994;120:20-36.

24. She K., Greated C.A., Easson W.J. Experimental study of three-dimensional breaking wave kinematics. Appl Ocean Res 1997;19:329-343.

25. Shemdin O.H. Wind-generated current and phase speed of wind waves. J Phys Oceanogr 1972;2:411-419.

26. Siddiqui M.H.K., Loewen M.R., Richardson C., Asher W.E., Jessup A.T. Simultaneous particle image velocimetry and infrared imagery of microscale breaking waves. Phys Fluids 2001;13:1891-1903.

27. Snyder R.L, Smith L., Kennedy R.M. On the formation of whitecaps by a threshold mechanism. Part III: Field experiment and comparison with theory. J Phys Oceanogr 1983;13:1505-1518.

28. Son S.Y., Kihm K.D. Evaluation of transient flow fields using digital cinematographic particle image velocimetry. Exp Fluids 2001;30:537-550.

29. Srokosz M.A. On the probability of wave breaking in deep water. J Phys Oceanogr 1986;16:382-385.

30. Stansell P., MacFarlane C. Experimental investigation of wave breaking criteria based on wave phase speeds. J Phys Oceanogr 2002;32:1269-1283.

31. Tulin MP., Li J.J. On the breaking of energetic waves. Int J Offshore Polar Eng 1992;2:46-53. 
32. Weissman M.A., Atakturk S.S., Katsaros K.B. Detection of breaking events in a wind-generated wave field. J Phys Oceanogr 1984;14:1608-1619.

33. Westerweel J. Efficient detection of spurious vectors in particle image velocimetry data. Exp Fluids 1994;16: 236-247.

34. Wright J.W., Keller W.C. Doppler spectra in microwave scattering from wind waves. Phys Fluids 1971;14:466-474.

35. Wu J. Wind-induced drift currents. J Fluid Mech 1975;68:49-70.

36. Wu C.H., Nepf H.M. Breaking criteria and energy losses for three-dimensional wave breaking. J Geophys Res 2002;107(C10):3177.

37. Xu D., Hwang P.A., Wu J. Breaking of wind-generated waves. J. Phys Oceanogr 1986; 16:2172-2178.

38. Zhang $X$. Surface image velocimetry for measuring short wind wave kinematics. Exp Fluids 2003;35:653-665. 
Table 1. Summary of experimental conditions and analyzed data

ase 1 ase 2 ase 3 ase 4 ase 5 ase 6

Experimental conditions

\begin{tabular}{|c|c|c|c|c|c|c|}
\hline$F(m)$ & 1.4 & 3.2 & 1.4 & 3.2 & 1.4 & 3.2 \\
\hline \multicolumn{7}{|l|}{$U(\mathrm{~m} / \mathrm{s})$} \\
\hline & .74 & .46 & 0.14 & 0.82 & 3.43 & 4.03 \\
\hline \multicolumn{7}{|l|}{$U_{*}(m / s)$} \\
\hline & .263 & .332 & .534 & .472 & .800 & .763 \\
\hline \multicolumn{7}{|l|}{$H_{s}(\mathrm{~cm})$} \\
\hline & .75 & .00 & .04 & .22 & .66 & .84 \\
\hline \multicolumn{7}{|l|}{$f_{p}(H z)$} \\
\hline & .23 & .24 & .54 & .82 & .09 & .58 \\
\hline \multicolumn{7}{|l|}{$c_{p}(\mathrm{~m} / \mathrm{s})$} \\
\hline & .48 & .70 & .61 & .86 & .75 & .99 \\
\hline \multicolumn{7}{|l|}{$U_{*} / c_{p}$} \\
\hline & .548 & .474 & .875 & .549 & .067 & .771 \\
\hline$g F / U_{*}^{2}$ & & & & & & \\
\hline & 615.2 & 062.7 & 91.8 & 020.5 & 746 & 90.5 \\
\hline
\end{tabular}

Frame transfer rate

(fps)

$\begin{array}{llllll}0 & 0 & 25 & 25 & 50 & 50\end{array}$

Shutter speed $\left(10^{-3} \mathrm{~s}\right)$

Number of runs

$\begin{array}{llll}5 & 5 & 5 & 5\end{array}$




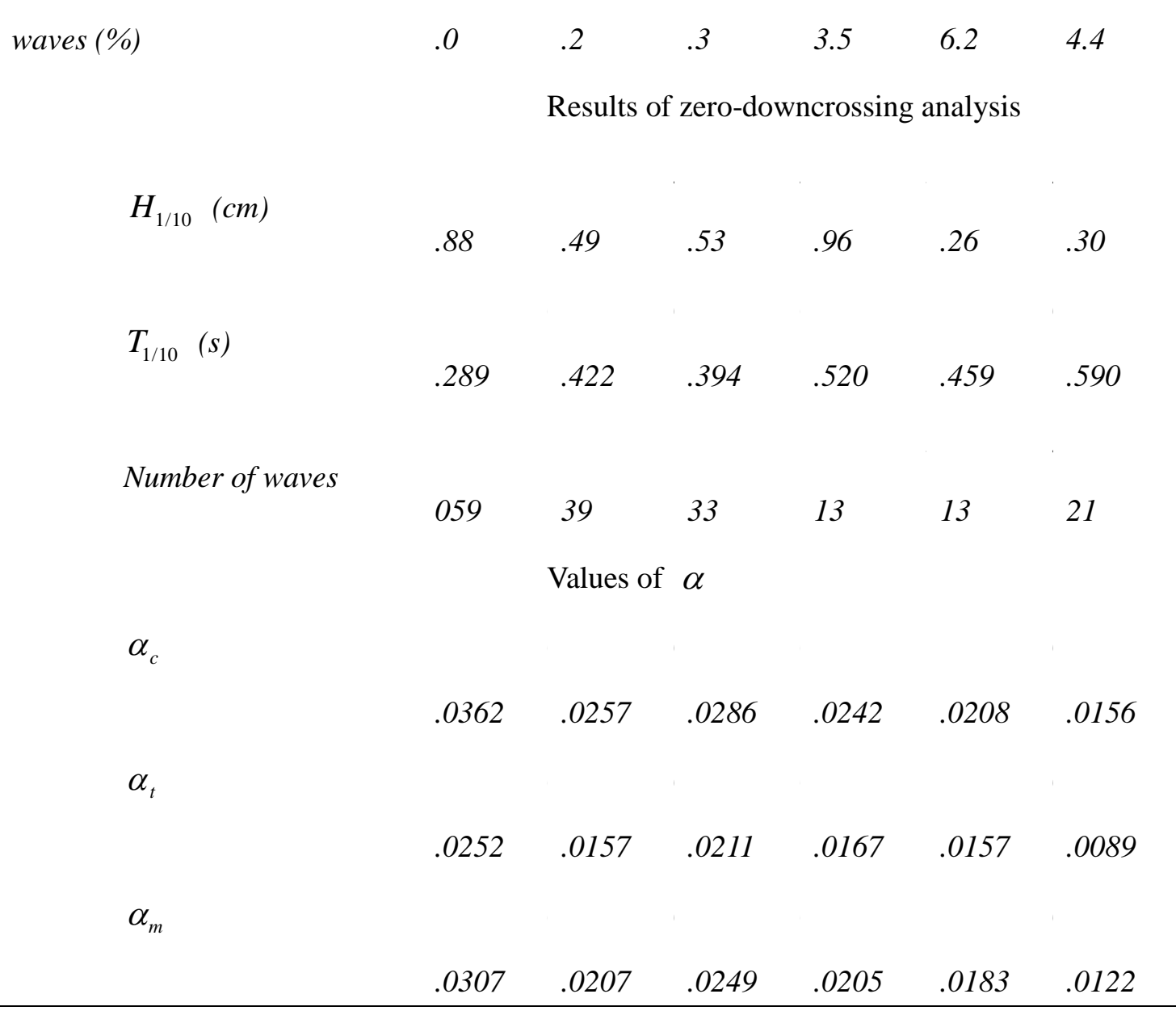




\section{Captions of Figures}

1. Definition of local wave shape parameters by Kjeldsen and Myrhaug [10].

2. Plan view of the wind wave flume.

3. Experimental setup: (a) Plan view and (b) Side view.

4. An sample image showing whitecaps near the wave crest (Case 6).

5. Wave phase speeds of the 20 selected waves at (a) crest and (b) trough.

6. An example of image processing: (a) Snapshot of a PIV image (Case 5). (b) Instantaneous velocity field. (c) Instantaneous acceleration field.

7. Mean values of the five geometric breaking criteria based on local wave shape parameters.

8. Values of $u_{s} / c$ of the 20 selected waves as a function of normalized time, $t / T$ : (a) Case

1, (b) Case 2, (c) Case 3, (d) Case 4, (e) Case 5, and (f) Case 6.

9. Mean values of $u_{s} / c$ for the six cases.

10. Values of $\langle u / c\rangle$ as a function of normalized depth, $z / H_{1 / 10}$ : (a) $t / T=1 / 4$ (wave trough) and (b) $t / T=3 / 4$ (wave crest).

11. Values of $a_{s} / g$ of the 20 selected waves as a function of normalized time, $t / T:$ (a) Case 1, (b) Case 2, (c) Case 3, (d) Case 4, (e) Case 5, and (f) Case 6.

12. Mean values of $a_{s} / g$ for the six cases.

13. Values of $\langle a / g\rangle$ as a function of normalized depth, $z / H_{1 / 10}$ : (a) $t / T=1 / 4$ (wave trough) and (b) $t / T=3 / 4$ (wave crest).

14. Mean values of wave breaking criteria as a function of $U_{*} / c_{p}$.

15. Mean values of wave breaking criteria as a function of $g F / U_{*}^{2}$. 


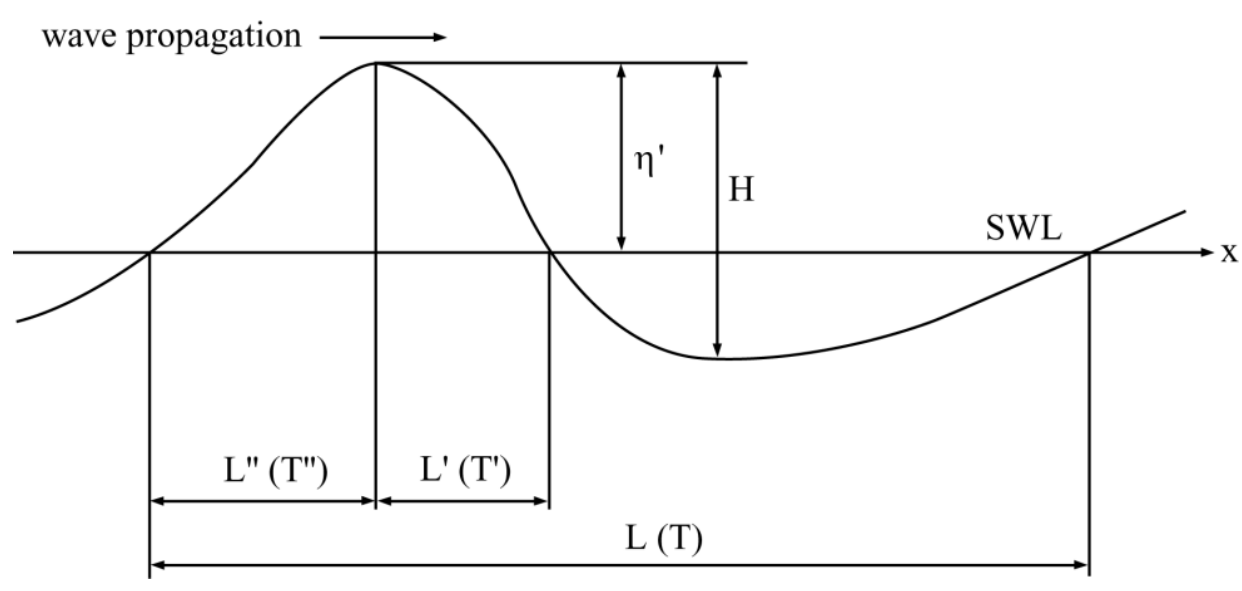

$\varepsilon=\frac{\eta^{\prime}}{L^{\prime}}=\frac{2 \pi \eta^{\prime}}{g T T^{\prime}} \quad \delta=\frac{\eta^{\prime}}{L^{\prime \prime}}=\frac{2 \pi \eta^{\prime}}{g T T} \quad \lambda=\frac{L^{\prime \prime}}{L^{\prime}}=\frac{T}{T^{\prime}} \quad \mu=\frac{\eta^{\prime}}{H}$

Fig. 1: Definition of local wave shape parameters by Kjeldsen and Myrhaug [10]. 


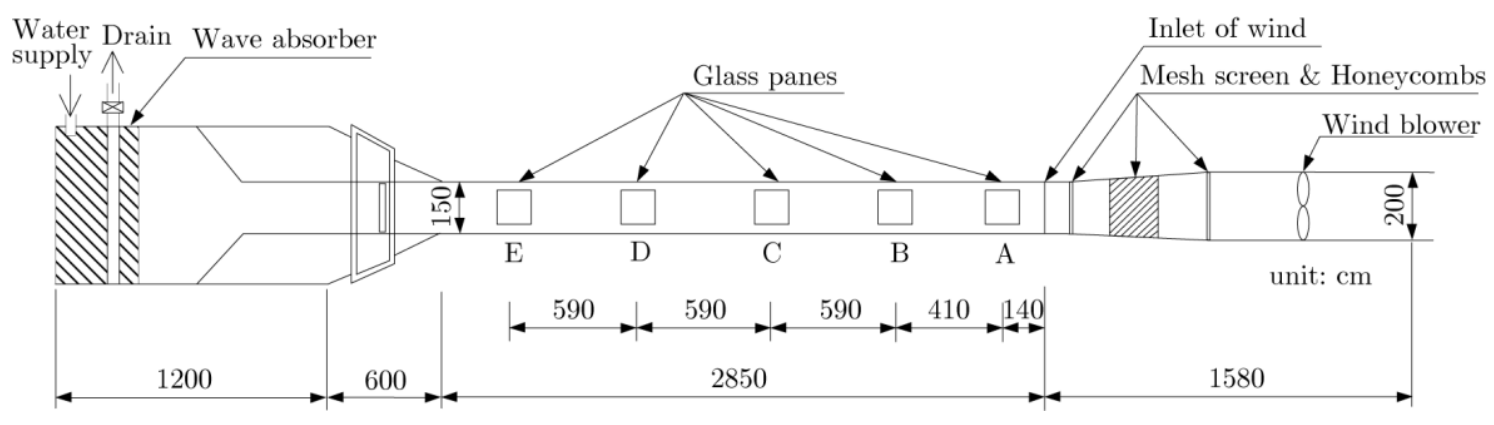

Fig. 2: Plan view of the wind wave flume. 

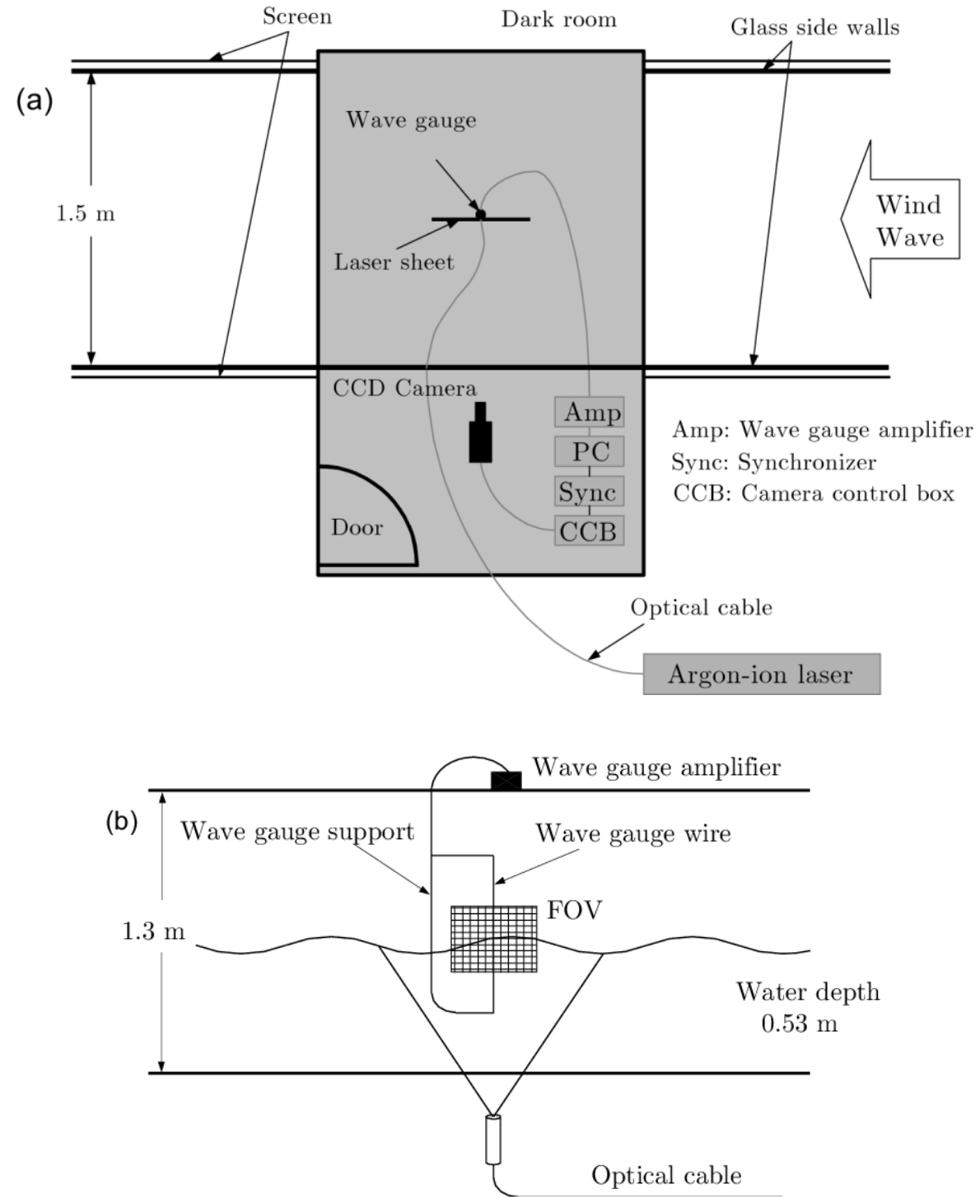

Fig. 3: Experimental setup: (a) Plan view and (b) Side view. 


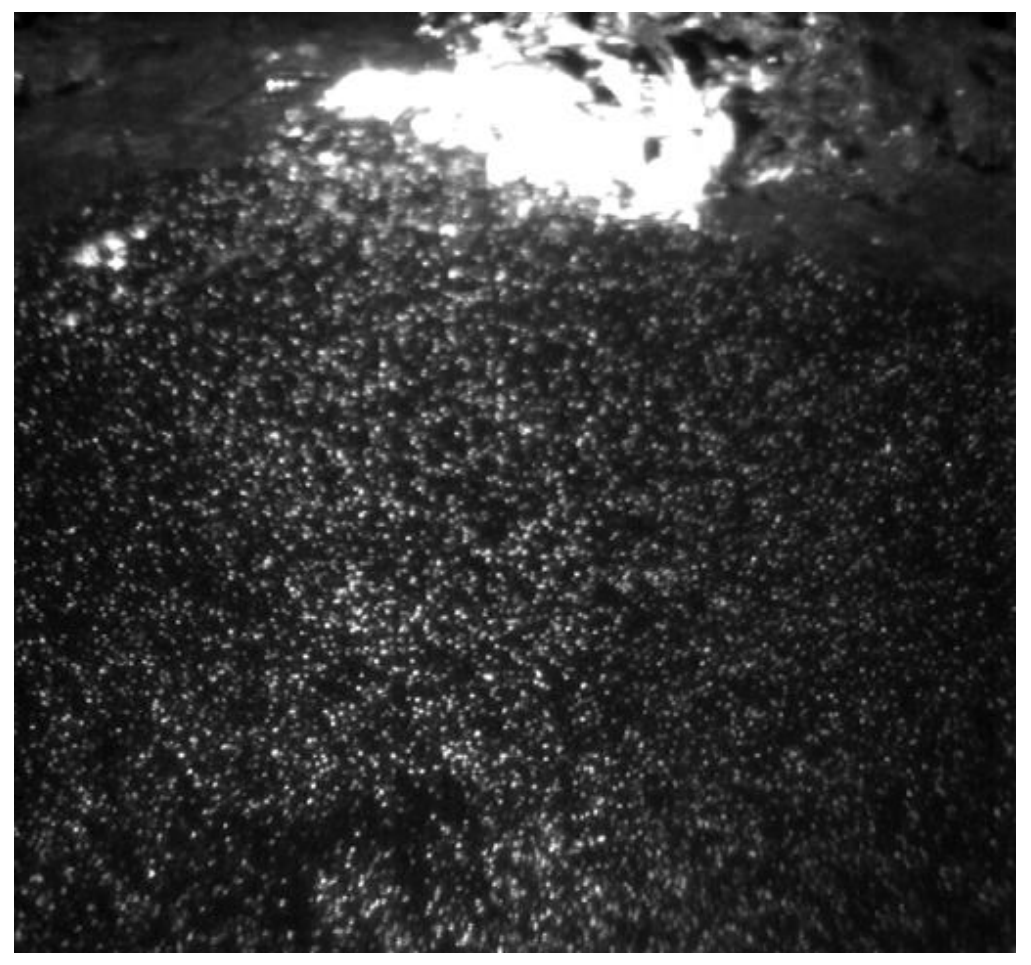

Fig. 4. A sample image showing whitecaps near the wave crest (Case 6). 

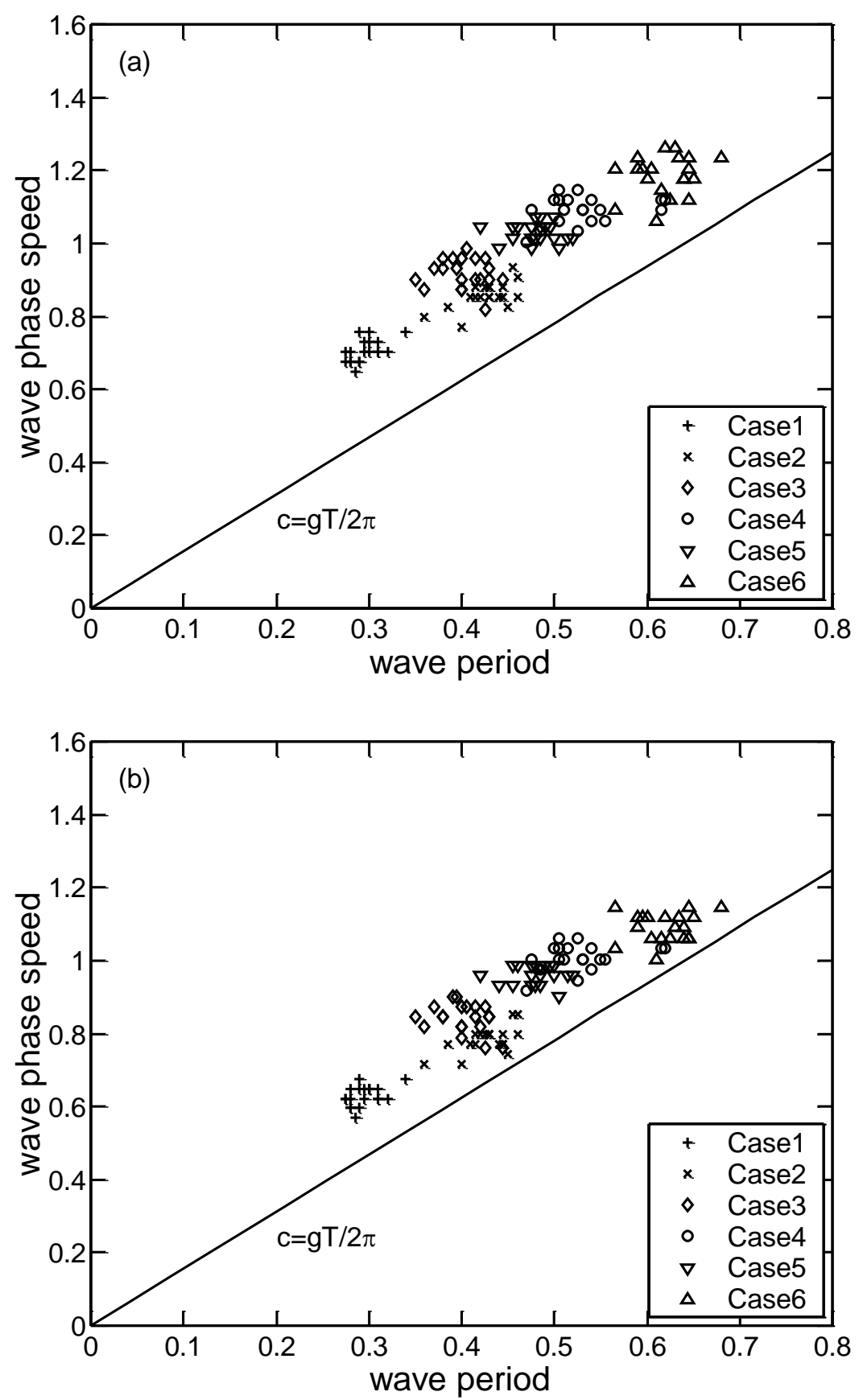

Fig. 5: Wave phase speeds of the 20 selected waves at (a) crest and (b) trough. 
(a)

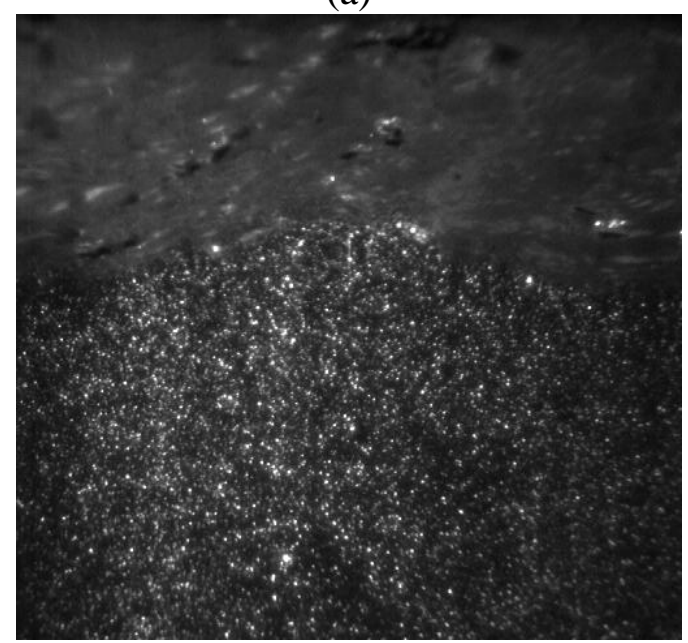

(b)

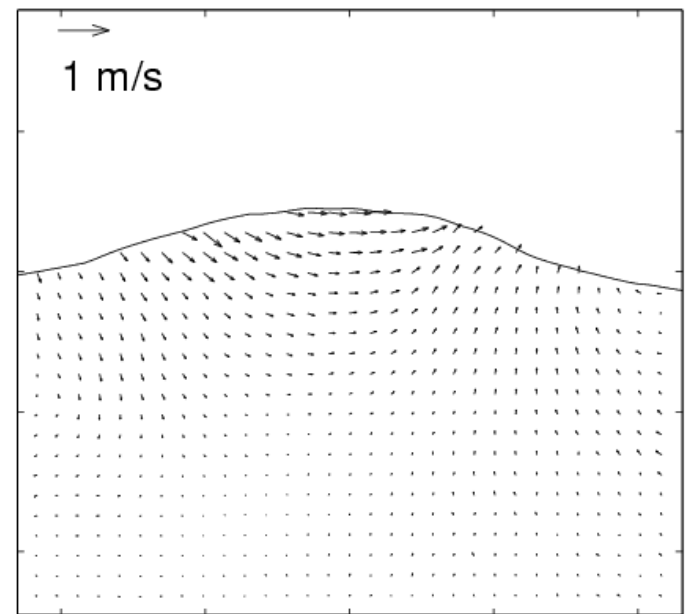

(c)

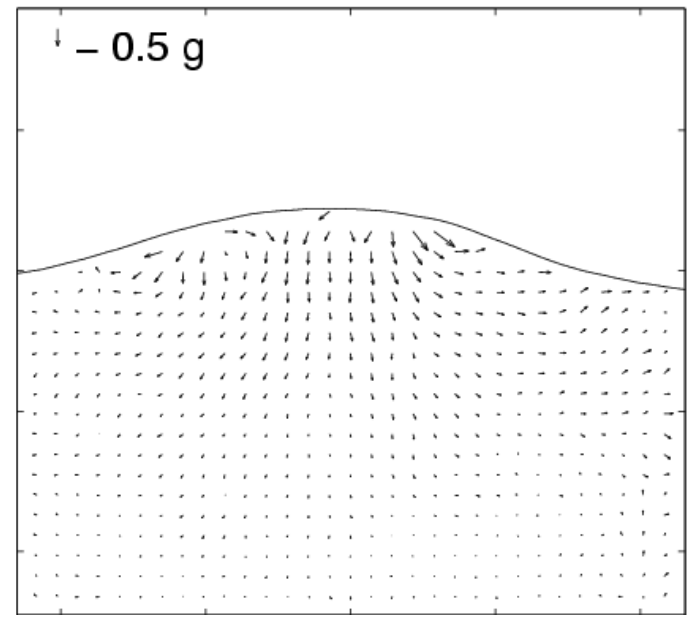

Fig. 6: An example of image processing: (a) Snapshot of a PIV image (Case 5). (b) Instantaneous velocity field. (c) Instantaneous acceleration field. 

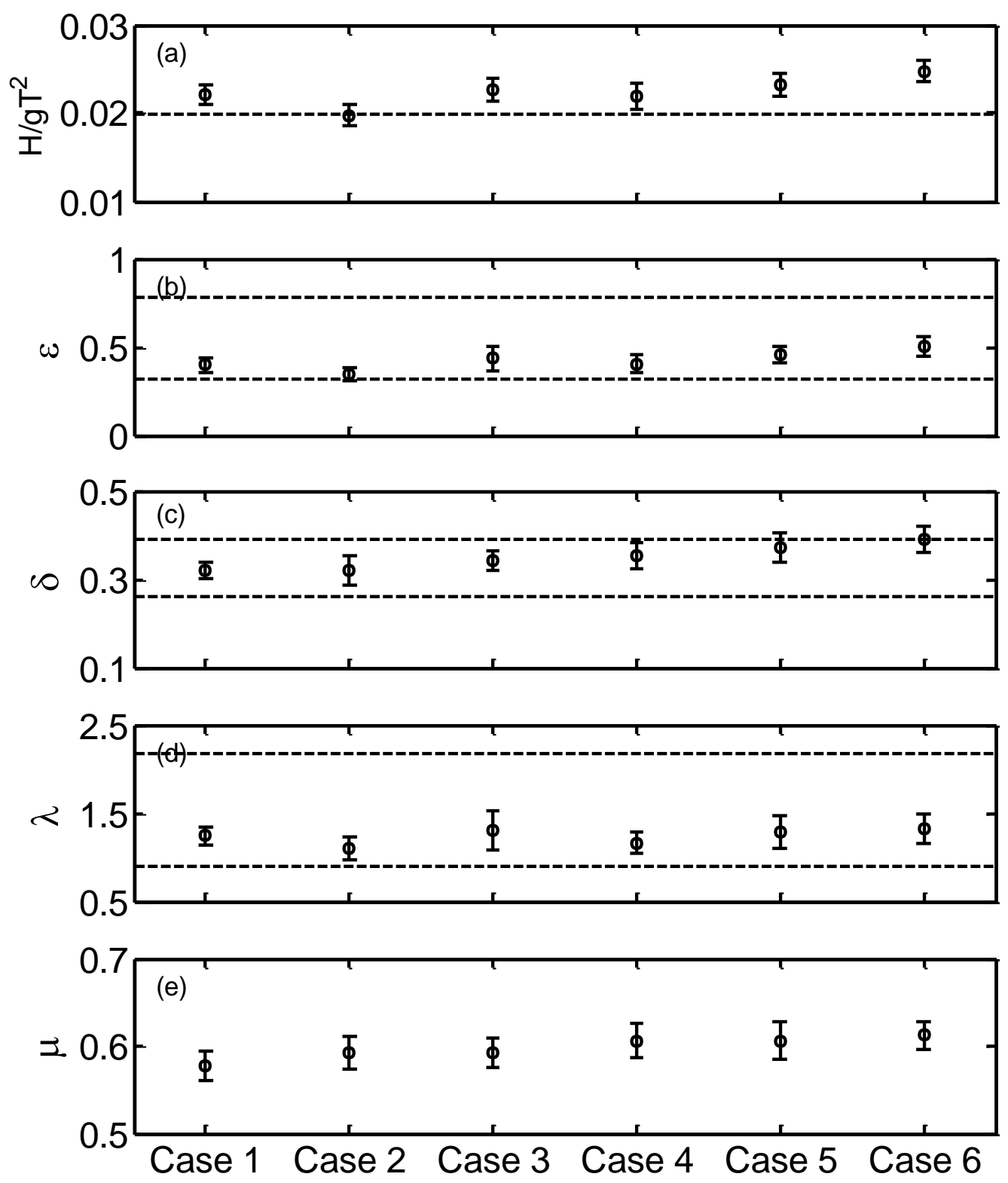

Fig. 7: Mean values of the five geometric breaking criteria based on local wave shape parameters. 

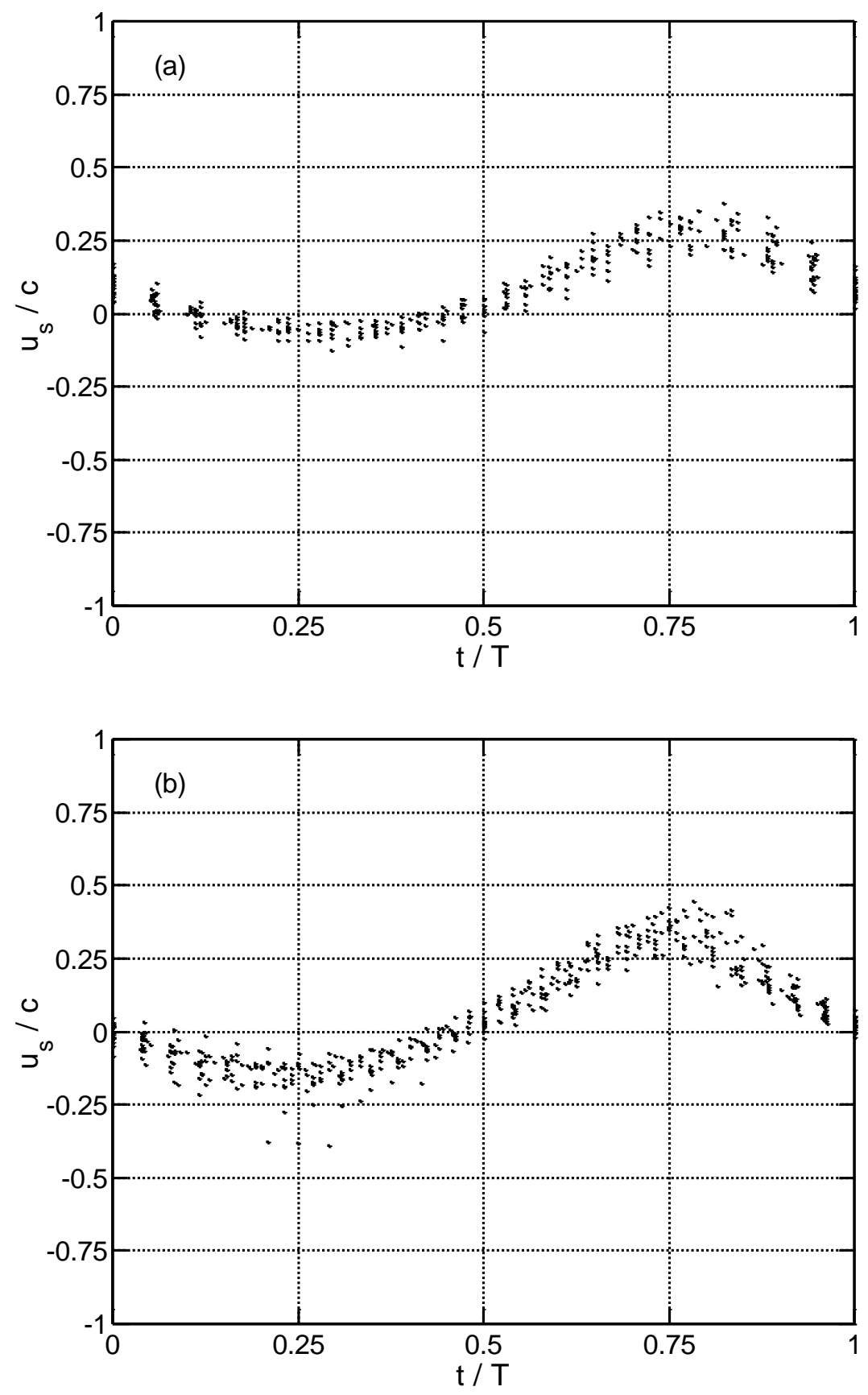

Fig. 8: Values of $u_{s} / c$ of the 20 selected waves as a function of normalized time, $t / T:$ (a) Case 1, (b) Case 2, (c) Case 3, (d) Case 4, (e) Case 5, and (f) Case 6. 

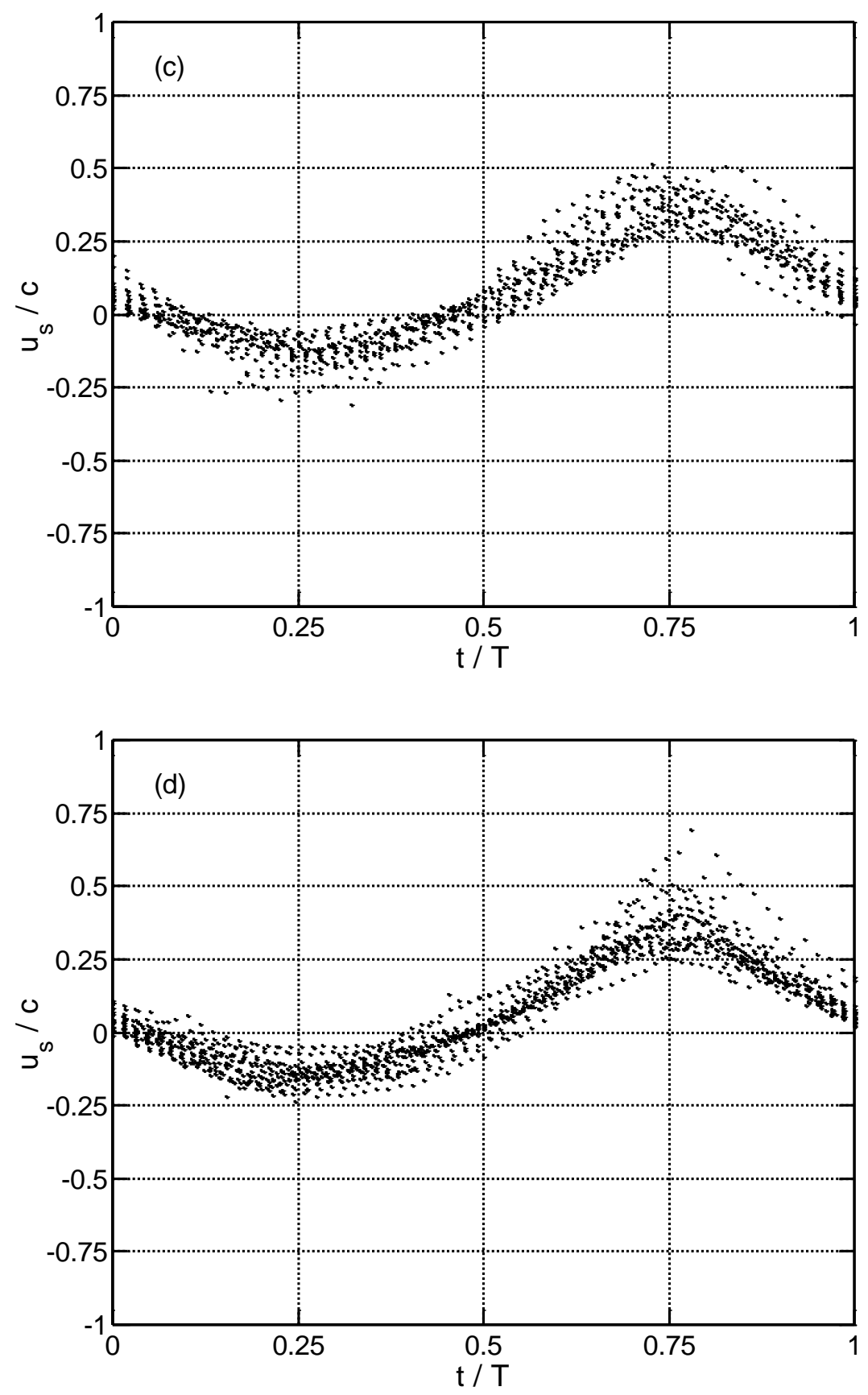

Fig. 8 (continued) 

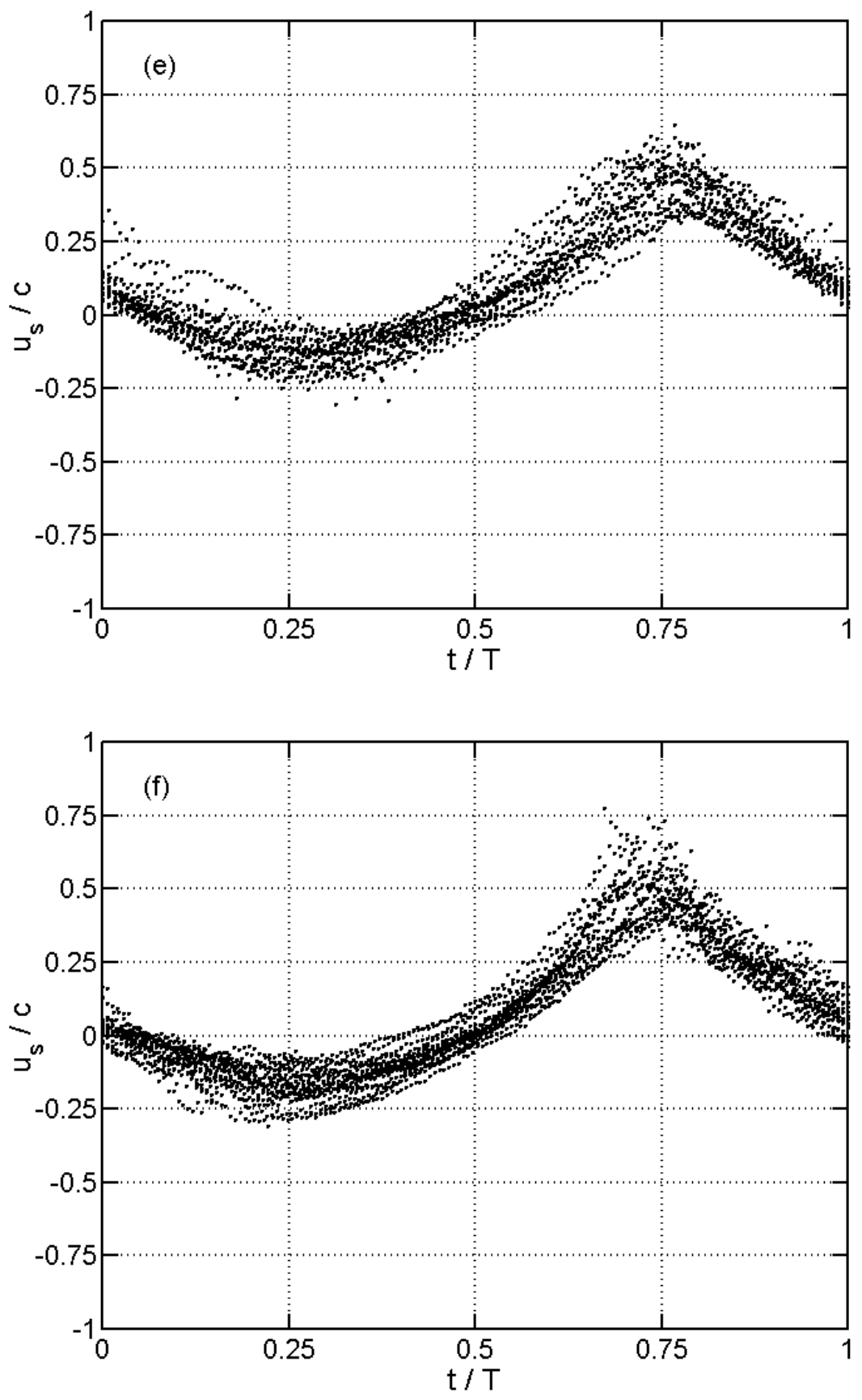

Fig. 8 (continued) 


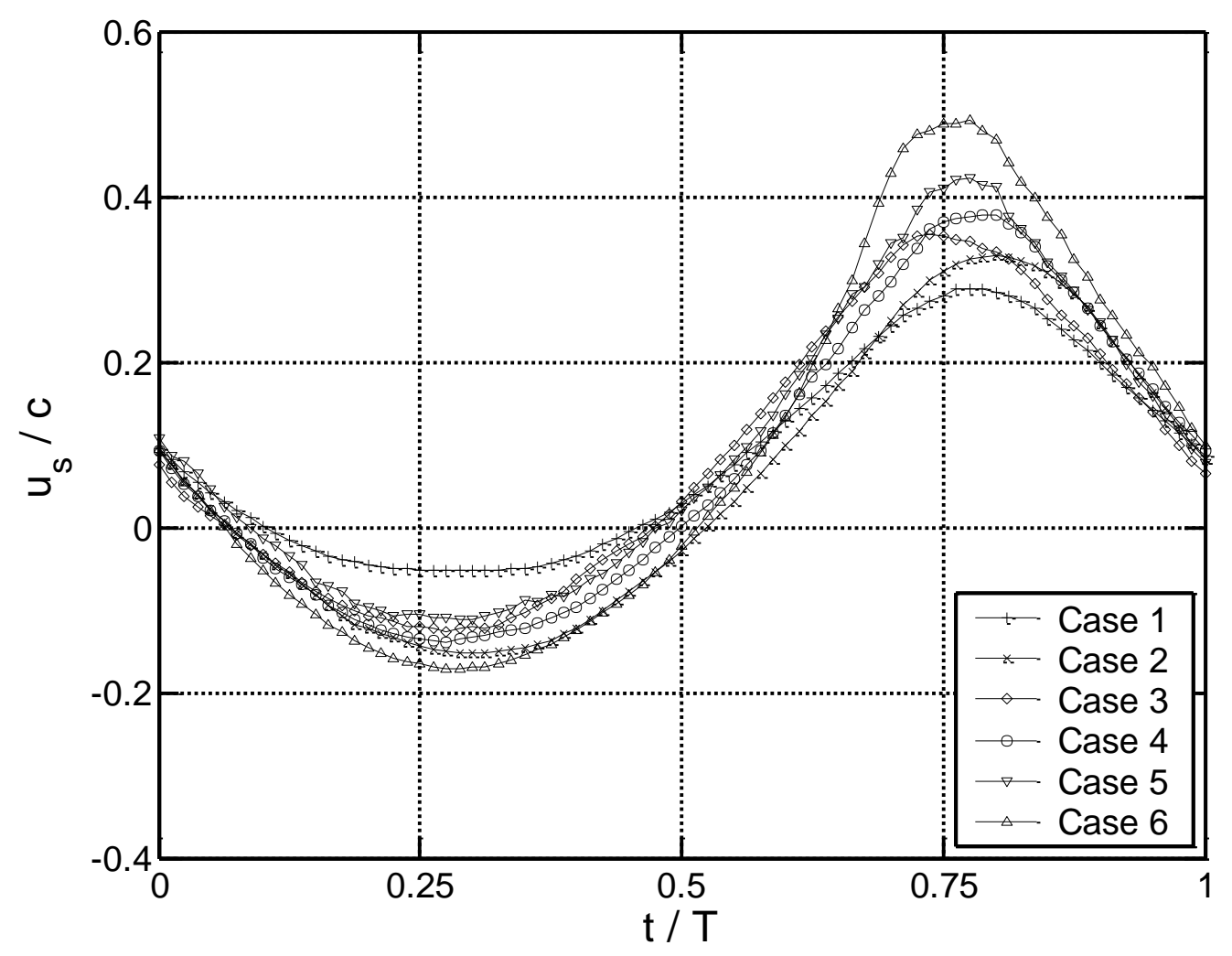

Fig. 9: Mean values of $u_{s} / c$ for the six cases. 

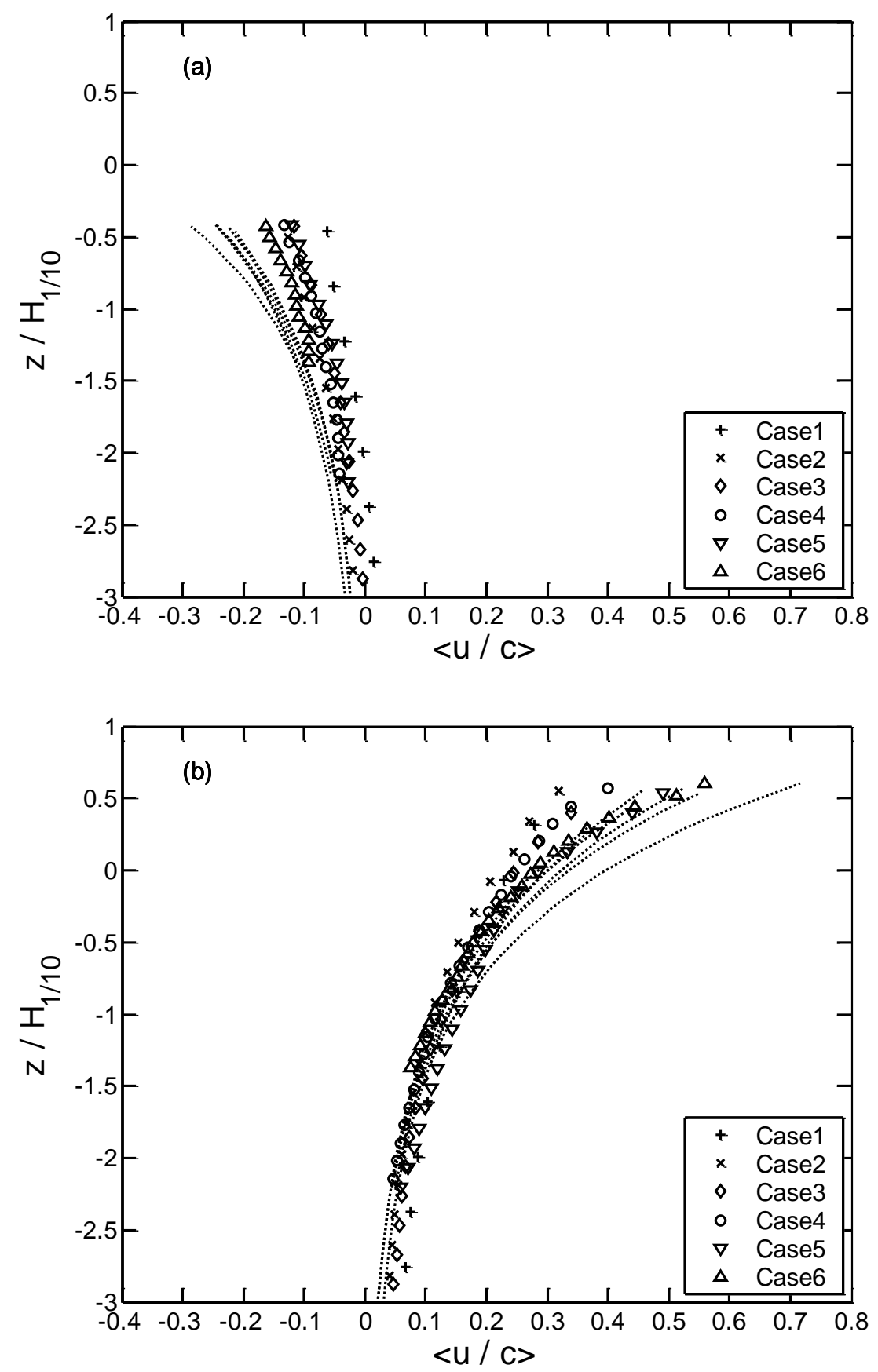

Fig. 10: Values of $\langle u / c\rangle$ as a function of normalized depth, $z / H_{1 / 10}$ : (a) $t / T=$ $1 / 4$ (wave trough) and (b) $t / T=3 / 4$ (wave crest). 

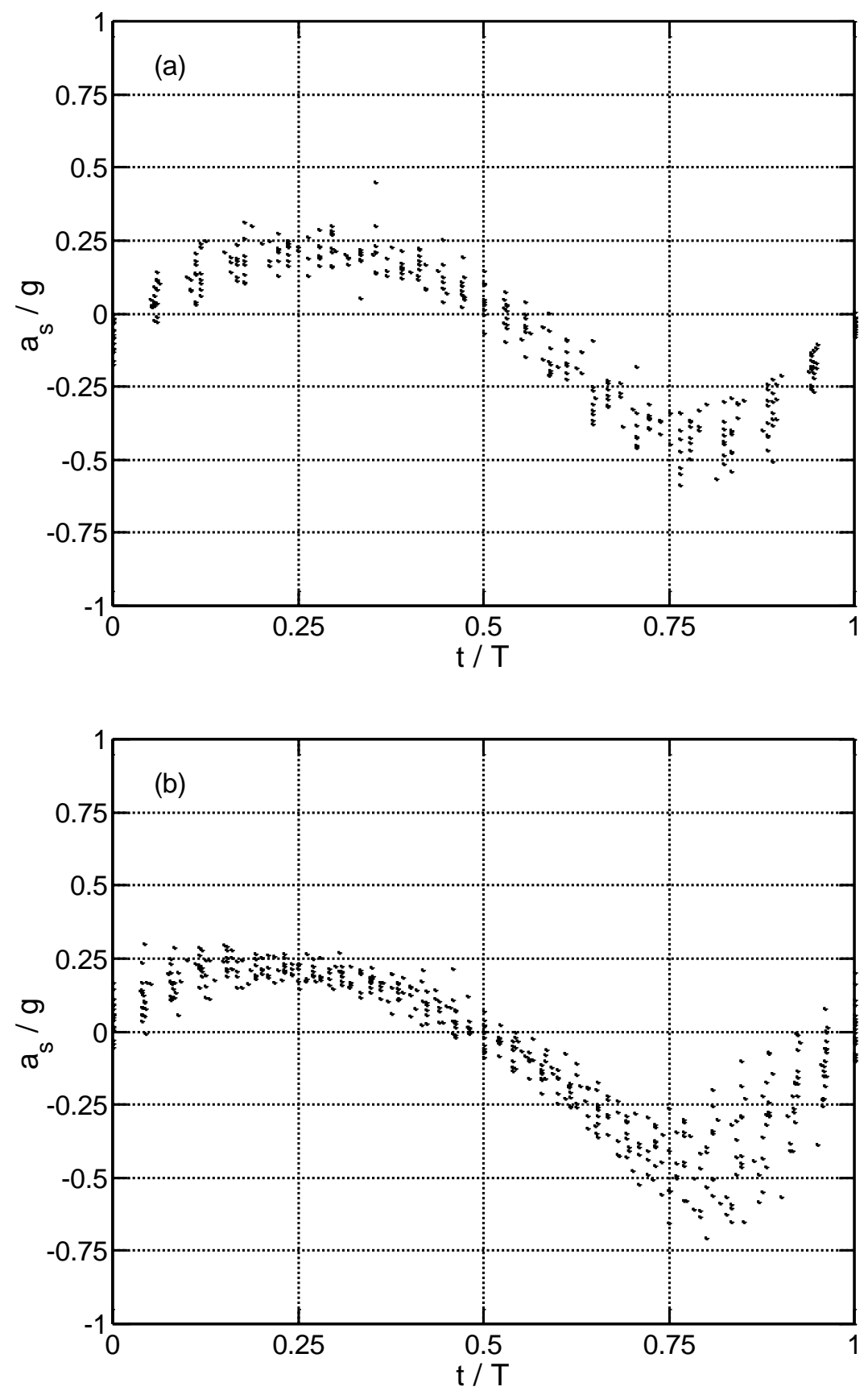

Fig. 11: Values of $a_{s} / g$ of the 20 selected waves as a function of normalized time, $t / T$ : (a) Case 1, (b) Case 2, (c) Case 3, (d) Case 4, (e) Case 5, and (f) Case 6. 

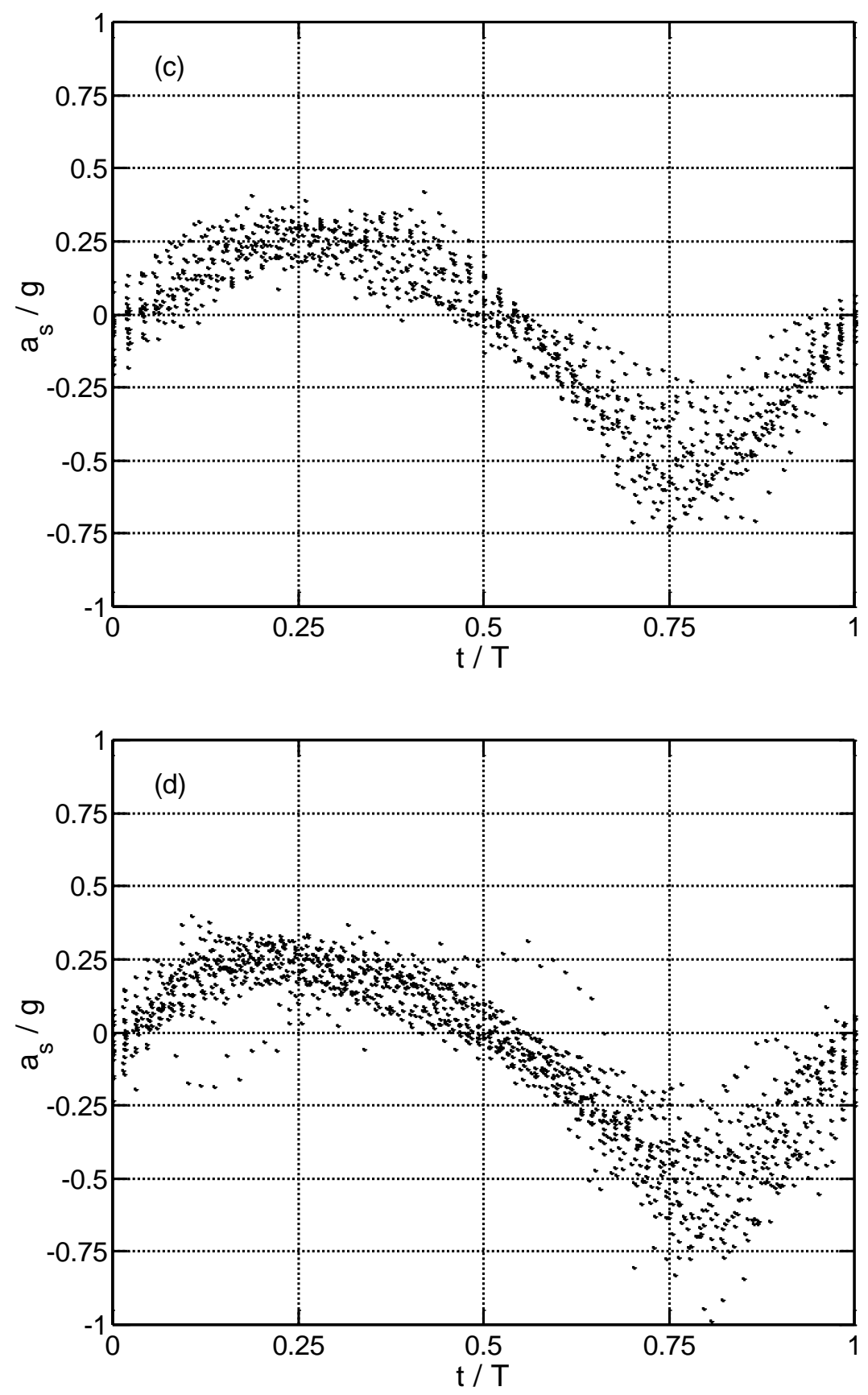

Fig. 11 (continued) 

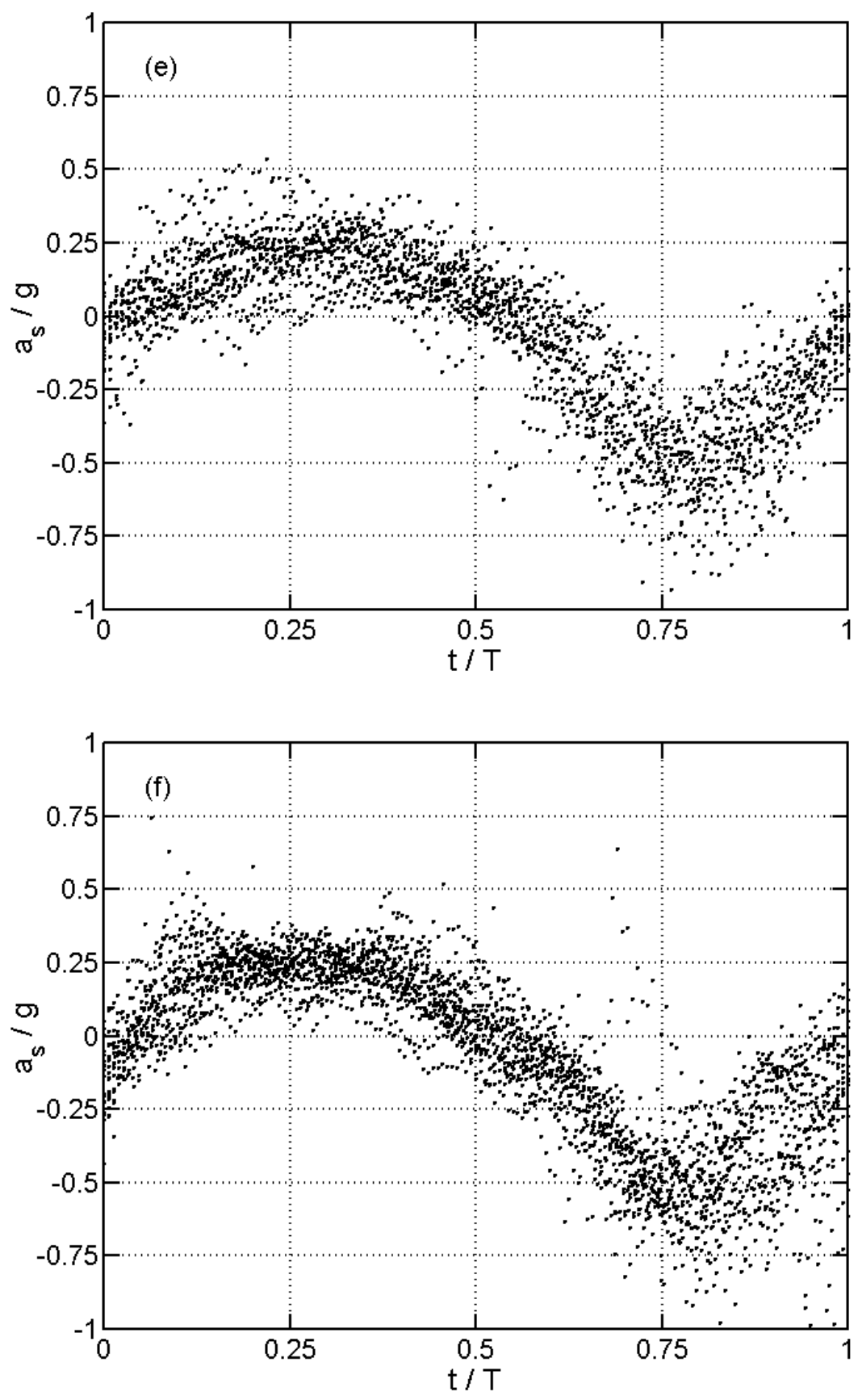

Fig. 11 (continued) 


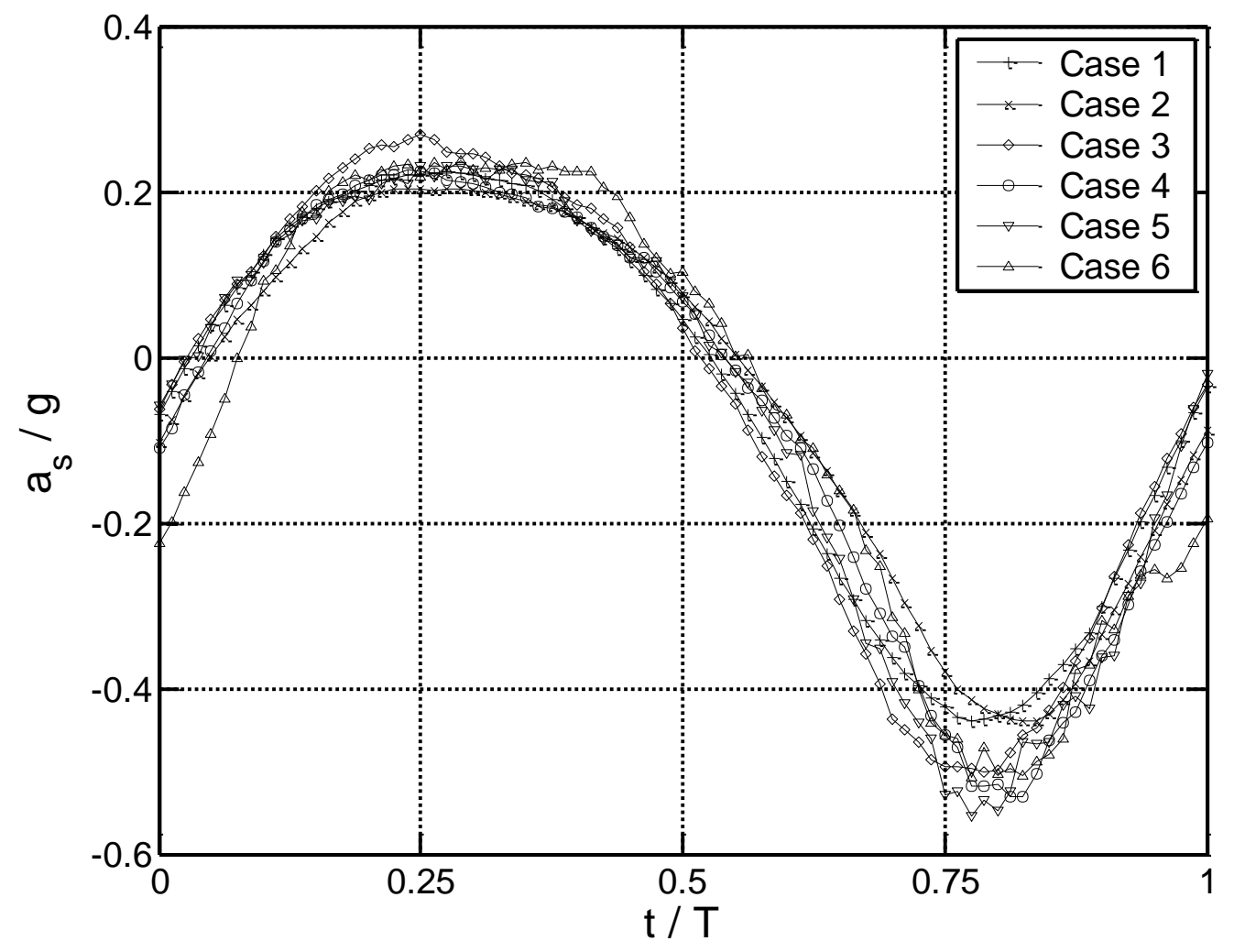

Fig. 12: Mean values of $a_{s} / g$ for the six cases. 

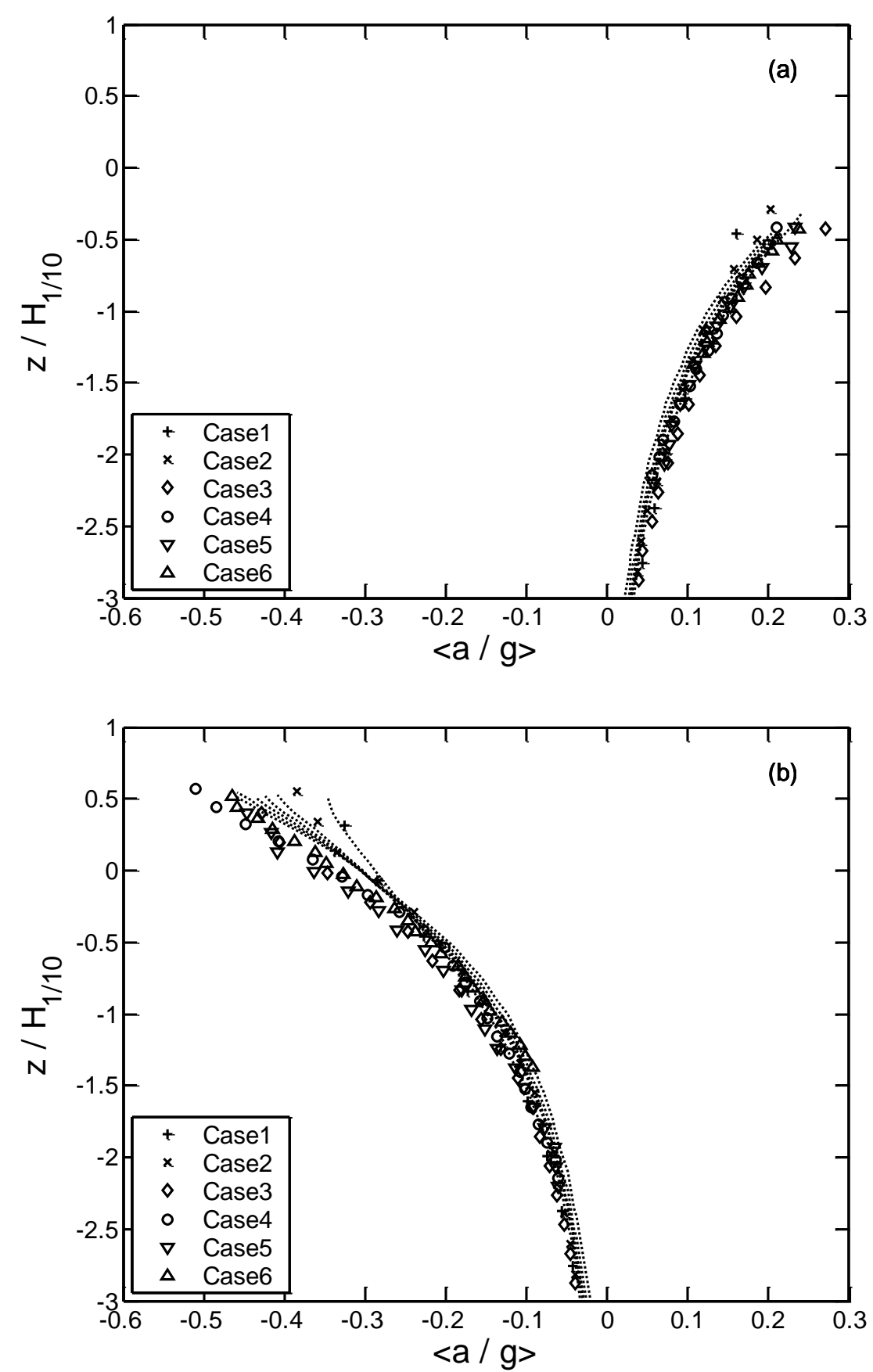

Fig. 13: Values of $\langle a / g\rangle$ as a function of normalized depth, $z / H_{1 / 10}$ : (a) $t / T=$ $1 / 4$ (wave trough) and (b) $t / T=3 / 4$ (wave crest). 


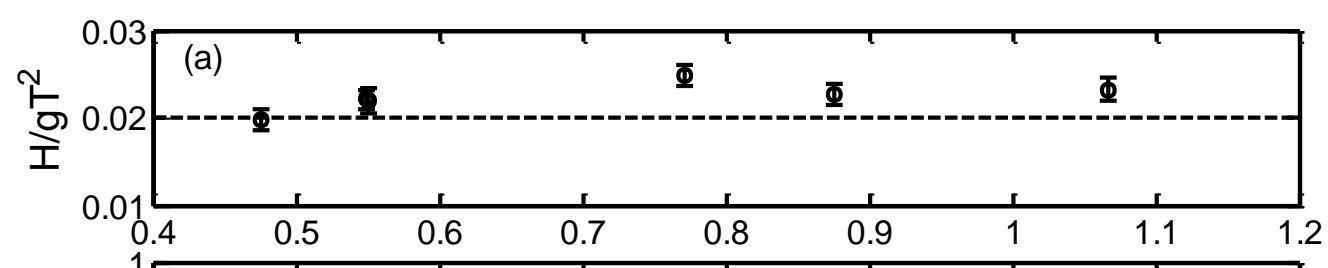

$\omega$
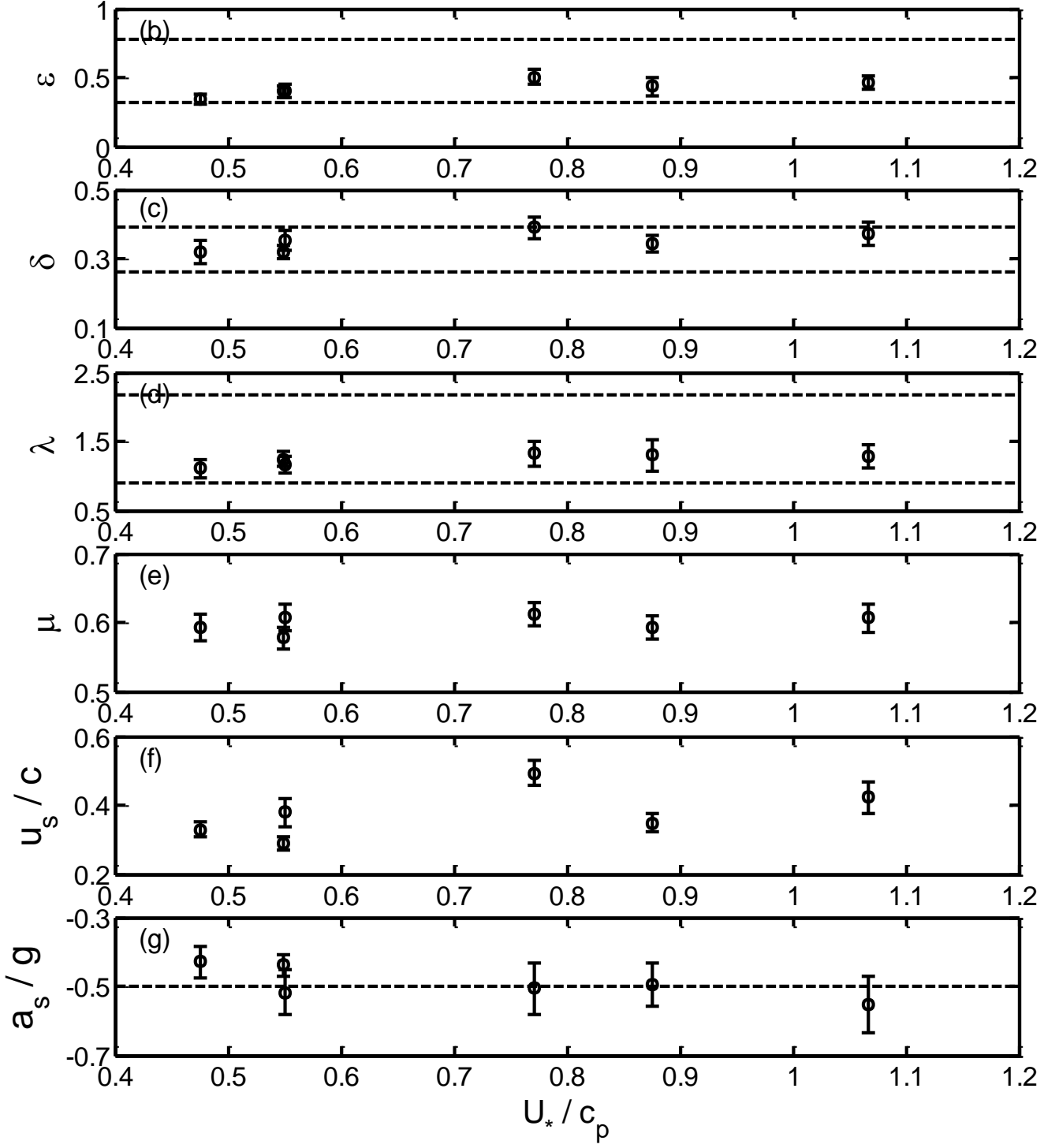

Fig. 14: Mean values of wave breaking criteria as a function of $U_{*} / c_{p}$. 

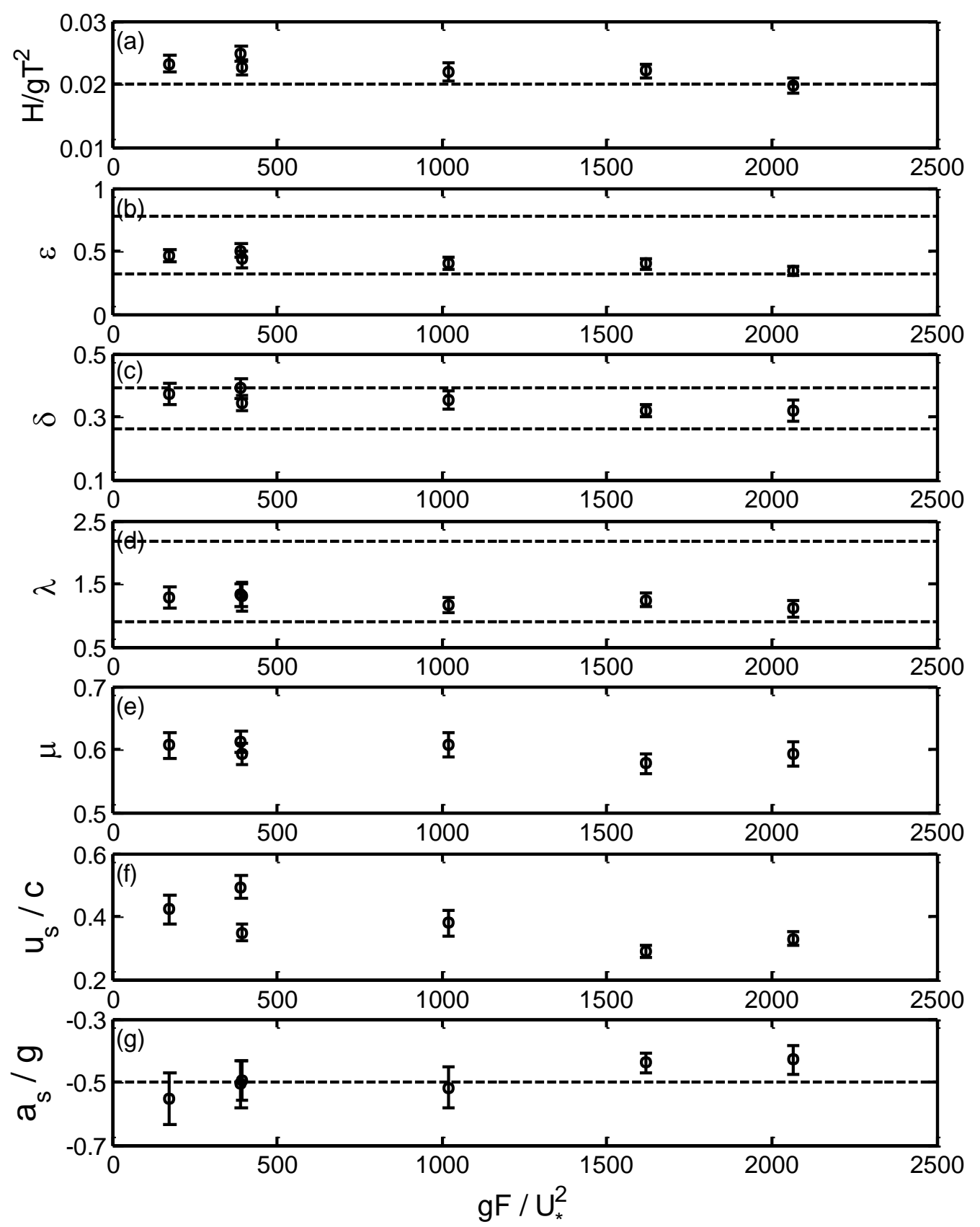

Fig. 15. Mean values of wave breaking criteria as a function of $g F / U_{*}^{2}$. 


\section{Answers to Comments Received}

First, we appreciate the reviewer's interest and comments on our manuscript. In the following, we provide responses to the reviewer's comments in a questionanswer format. The answers are typed in italic.

Review report on a paper entitled "Experimental investigation of breaking criteria of deep water wind waves under strong wind action" by Sang Ho Oh et al.

This paper addresses a topic that will be of interest to readers of AOR, concerning results from experimental investigations of breaking criteria of deep water waves under strong wind action. They find that the previously suggested limit value of $0.5 \mathrm{~g}$ for the vertical acceleration beneath the wave crest can be used for discriminating breaking waves under strong wind action. They also demonstrate that geometric and kinematic breaking criteria are not capable of explaining the occurrence of breaking. The results are presented in a generally clear and logical manner. We consider that the paper will be suitable for publication in AOR, subject to the consideration by the authors of the following points.

1. p.9, 1st paragraph of 3.2.1: Give the range of the measuring elevations. It was stated in the first paragraph of Section 3.2.1 as the reviewer suggested.

2. p.10, 1st paragraph: Make a statement of how accurate the main experiment was relative to the preliminary experiments, Explain how $U_{*}$ was calculated; from the best fit by log-profile, or...?

The preliminary experiment was carried out in order to obtain reliable estimates of wind and wave parameters prior to conducting the main experiment because the measurement time of the main experiment was not long due to the restriction of the synchronized PIV system. The first paragraph of section 3.2.1 was restated by adding this explanation. The values of $U_{*}$ was calculated by assuming a logarithmic wind profile. This was mentioned in the first paragraph of section 
3. Section 4.1; Is it possible to be more specific about the type of breaking waves, spilling and/or plunging breaker?

Spilling-type breaking waves were observed in the experiment. It was stated in the last paragraph of section 4.1 as the reviewer suggested.

4. Section 5.1; When using the inverse wave age the reader should be made clearly aware of the meaning of this by the authors. The wave age $U_{*} / c_{p}$, which for wind waves are taken as less than 40 (according to Toba et al. (1990), J.Physical Oceanography 20,705-721). Low value represents young waves and high value represents closer to fully developed waves. An affirmative wave age parameter for in-situ conditions is often used by replacing $U_{*}$ by $U_{10}$ ( referring to the $10 \mathrm{~m}$ elevation ). Realistic wave age limits are often taken as $0.03 \leq c_{p} / U_{10} \leq 1.0$ (Toba et al (1990)). If $U_{10}$ is replaced by (referring to the $40 \mathrm{~cm}$ elevation ), then $c_{p} / U_{10}$ is in the range of 0.06 to 0.09 , and with $c_{p} / U_{*}$ in the range 0.9 to 2.1 , suggesting that the waves represent very young waves. It is suggested that the authors consider to include the wave age in Table 1. The meaning of the inverse wave age, $U_{*} / c_{p}$ is the wind forcing to the underlying waves, as stated in the first paragraph of section 5.4. We used this parameter since the inverse wave age is a more proper parameter than the wave age, $c_{p} / U_{*}$ for representing the relative magnitude of the wind speed. We included the values of $U_{*} / c_{p}$ in Table 1 , from which the values of $c_{p} / U_{*}$ are readily calculated. We briefly mentioned in section 5.4 (p. 26) that the waves are very young waves. We added a section 5.4 in order to discuss the correlation between the wave breaking criteria and $U_{*} / c_{p}$ as well as the nondimensional fetch, $g F / U_{*}^{2}$. According to this change, Section 5.1 was restated and Fig. 7 was redrawn. 
5. Section 5.2; Here the fetch $(F)$ is also discussed. Did the authors consider if there exists any correlation between the results and the nondimensional fetch, e.g. $g F / U^{2}$ ? Did the authors make any attempts to find out if any existing wave theory can be used to predict the behavior observed in Fig. 10?

We included the values of the nondimensional fetch, $g F / U_{*}^{2}$ in Table 1 and discussed the relationship with the breaking criteria in a new section 5.4, as mentioned in the above answer. We compared the results shown in Fig. 10 with the velocity profiles of the linear wave theory normalized by the measured phase speed, calculated by Eq. (1). We provided explanation on this in the last paragraph of Section 5.2. In addition, we compared the measured acceleration profiles shown in Fig. 13 with the theoretical ones of Longuet-Higgins (1986) and provided explanation in the last paragraph of Section 5.3. According to this change, some sentences in the previous paragraph were removed.

6. p.31; Check if Ref.18 is referred to in the text.

We removed it from the reference list.

Other corrections not commented by the reviewer

1) Figs. 9 and 12 were redrawn to correct a mistake in taking the mean values of $u_{s} / c$ and $a_{s} / g$. The overall trend of the new figures are nearly the same as the old ones, but locally slightly different.

2) We removed two sentences in the third paragraph of section 5.3 because the values of $t / T$ that correspond to wave crests are not always exactly identical to $t / T=0.75$ among the test cases, but may slightly deviate from $t / T=0.75$ depending on the test cases.

3) The citation of the reference [18] (Peirson and Banner, 2003) was slightly modified (page 6 and 26) according to private communication with one of the authors of the reference. 